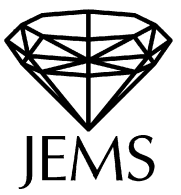

G. Alberti $\cdot$ S. Baldo $\cdot$ G. Orlandi

\title{
Functions with prescribed singularities
}

Received March 18, 2002 / final version received May 9, 2003

Published online July 10, 2003 - (C) Springer-Verlag \& EMS 2003

\begin{abstract}
The distributional $k$-dimensional Jacobian of a map $u$ in the Sobolev space $W^{1, k-1}$ which takes values in the the sphere $S^{k-1}$ can be viewed as the boundary of a rectifiable current of codimension $k$ carried by (part of) the singularity of $u$ which is topologically relevant. The main purpose of this paper is to investigate the range of the Jacobian operator; in particular, we show that any boundary $M$ of codimension $k$ can be realized as Jacobian of a Sobolev map valued in $S^{k-1}$. In case $M$ is polyhedral, the map we construct is smooth outside $M$ plus an additional polyhedral set of lower dimension, and can be used in the constructive part of the proof of a $\Gamma$-convergence result for functionals of Ginzburg-Landau type, as described in [2].
\end{abstract}

Key words. Jacobian - Sobolev maps - singular maps - integral currents - rectifiability dipole construction - complete intersections - Brouwer degree - coarea formula - GinzburgLandau functionals

\section{Introduction}

Given a map $u=\left(u_{1}, u_{2}\right)$ from a domain $\Omega \subset \mathbb{R}^{2}$ into $\mathbb{R}^{2}$ which belongs to the Sobolev class $W^{1,2}$, the Jacobian determinant of $u$ is $J u:=\operatorname{det}(D u)$ (notice the absence of modulus). This pointwise definition may not give an $L^{1}$-function for less regular maps $u$, but the Jacobian can be still defined in some cases using the identity

$$
J u=\frac{\partial}{\partial x_{1}}\left(u_{1} \frac{\partial u_{2}}{\partial x_{2}}\right)-\frac{\partial}{\partial x_{2}}\left(u_{1} \frac{\partial u_{2}}{\partial x_{1}}\right) .
$$

Indeed, the right-hand side of (1.1) makes sense, as a distribution, for all $u$ of class $W^{1,1} \cap L^{\infty}$; the two definitions clearly agree for maps of class $W^{1,2}$.

G. Alberti: Dipartimento di Matematica, Università di Pisa, via Buonarroti 2, 56127 Pisa, Italy, e-mail: alberti@dm.unipi.it

S. Baldo: Dipartimento di Matematica, Università di Trento, via Sommarive 14, 38050 Povo (Trento), Italy, e-mail: baldo@science.unitn.it

G. Orlandi: Dipartimento di Informatica, Università di Verona, Strada le Grazie 15, 37134 Verona, Italy, e-mail: orlandi@sci.univr.it

Mathematics Subject Classification (2000): 46E35 (53C65, 49Q15, 26B10, 58A25) 
If we restrict our attention to maps $u$ which take values in the unit circle $S^{1}$, for smooth $u$ the area formula implies that $J u$ vanishes, because $S^{1}$ has null twodimensional measure. In fact, the same holds for maps of class $W^{1, p}$ when $p \geq 2$, but is no longer true when $p<2$ : for example, the (distributional) Jacobian of $u(x):=x /|x|$ defined by $(1.1)$ is $\pi \delta_{0}$, where $\delta_{0}$ is the Dirac mass centered at the origin, while $\operatorname{det}(D u)$ is almost everywhere null. More generally, if $u$ is smooth except for finitely many singular points $x_{i}$, then $J u$ is a sum of Dirac masses centered at $x_{i}$, endowed with a multiplicity that can be recovered from the degree of the restriction of $u$ to any curve which winds around $x_{i}$ (see Sect. 3.6 below). The distributional Jacobian was first applied in the context of nonlinear elasticity by J. Ball in [5] (see also [31]), while the relation with topological singularities and Dirac masses was pointed out by H. Brezis, J.-M. Coron, and E. Lieb [14] in the context of harmonic maps and liquid crystal theory.

For domains and target spaces of general dimension, the situation is quite similar. Given a regular map $u=\left(u_{1}, \ldots, u_{k}\right)$ from $\Omega \subset \mathbb{R}^{n}$ into $\mathbb{R}^{k}$, it is convenient to define the $k$-dimensional Jacobian as the pull-back according to $u$ of the standard volume form on $\mathbb{R}^{k}$, that is, $J u:=d u_{1} \wedge \cdots \wedge d u_{k}$. This definition makes sense for all maps of class at least $W^{1, k}$, and can be extended to maps of class $W^{1, k-1} \cap L^{\infty}$ using the identity $J u=\frac{1}{k} d\left(\sum(-1)^{i-1} u_{i} \bigwedge_{j \neq i} d u_{j}\right)$. As before, if $u: \Omega \rightarrow S^{k-1}$ is of class $W^{1, p}$ with $p \geq k$, then $J u=0$, but this may be no longer true when $p<k$. More precisely, if $u$ is smooth outside a regular surface $M$ of codimension $k$, then $J u$ is supported on the set $M$, and can be reconstructed from $M$ and the degree of the restriction of $u$ to any surface of dimension $k-1$ which winds around $M$ (cf. [28] and $\$ 3.7$ below). In this sense, we may say that $J u$ represent the part of the singularity of $u$ which is topologically relevant ${ }^{1}$.

The previous observations on maps with regular singularity suggest the following general structure theorem (see [27], [28]): if $u: \Omega \rightarrow S^{k-1}$ is of class $W^{1, k-1}$ and $J u$ is a locally bounded measure, then $J u$ is concentrated on a set of (Hausdorff) codimension $k$, and more precisely it can be identified ${ }^{2}$ with a rectifiable current of codimension $k$ and without boundary. This result can also be obtained as a corollary of Federer-Fleming boundary rectifiability theorem and the following slightly more general statement (see Theorem 3.8 below): the Jacobian of a map $u: \Omega \rightarrow S^{k-1}$ of class $W^{1, k-1}$ agrees with the boundary of a rectifiable current of codimension $k-1$. For $k=2,4,8$ this theorem was proved independently in [32], using results from [21]; a similar statement for maps in the trace space $W^{1-1 / k, k}$ was given in [23] for any $k$.

The distributional Jacobian of maps in $W^{1, k-1}\left(\Omega, S^{k-1}\right)$ is particularly interesting because is the obstruction to strong approximation by smooth maps (see [6], [7],

1 This statement must be taken with care: if $h: S^{3} \rightarrow S^{2}$ is the Hopf map - namely the one that generates the third homotopy group of $S^{2}-$ and $u: \mathbb{R}^{4} \rightarrow S^{2}$ is given by $u(x):=h(x /|x|)$, then $u$ belongs to $W^{1, p}\left(\Omega, S^{2}\right)$ for every $p<3$, and in particular $J u=0$; yet the singularity at the origin cannot be discarded as topologically unrelevant (cf. [24]).

2 The Hodge-type $\star$ operator defined in Sect. 2.7 provides a canonical identification of $k$-covectors and $(n-k)$-vectors; thus $\star J u$ is an $(n-k)$-current, although in general not a rectifiable one. In the rest of this introduction we tacitly assume this identification. 
and also [32], [33], [34], where the Jacobian is known as topological singularity). In fact, $J u$ is just the projection of the boundary of the graph of $u$, which plays an essential rôle in the theory of cartesian currents developed by M. Giaquinta, G. Modica, and J. Souček ([19], [20], [21]); in particular, the rectifiability of $J u$ can be recovered by the product structure of this boundary (cf. Remark 7.10). More recently, R.L. Jerrard and H.M. Soner [28] proposed a systematic study of the distributional Jacobian of maps valued in $\mathbb{R}^{k}$ which underlines the analogy with the theory of $B V$ functions.

The main purpose of this paper is to investigate which surfaces, or currents, can be obtained as Jacobians of $S^{k-1}$-valued maps, that is to say, to construct maps with a prescribed singularity. Our main result in this direction is the exact converse of Theorem 3.8:

Main result (see Theorem 5.6). Let $\Omega$ be an open set in $\mathbb{R}^{n}$, and let $M$ be the boundary of a rectifiable current of codimension $k-1$ in $\Omega$. Then there exists a map $u \in W^{1, k-1}\left(\Omega, S^{k-1}\right)$ whose Jacobian agrees, up to a canonical identification, with the current $M$.

The proof of this result is based on a dipole construction much in the spirit of [14], and a careful iteration argument. In Sect. 6.7 we sketch a very simple proof for the case $k=2$ based on the theory of $B V$ functions.

Note that the previous statement, although very general, does not settle the question completely. In particular, it is natural to ask how regular can be $u$ when $M$ is a regular surface. In this direction, we can show that when $M$ is a smooth surface of codimension $k=2$, then $u$ can be taken of class $W_{\text {loc }}^{1, p}$ for every $p<1^{*}=n /(n-1)$ and smooth in the complement of $M$ (see Theorem 4.4 and Sect. 6.4).

Such a result could not be proved for surfaces of codimension $k>2$ : as pointed out in Sect. 6.5, constructing a map $u$ smooth in the complement of $M$ is quite close to proving that $M$ is the boundary of a smooth surface (and not just of a rectifiable current) and even a complete intersection, and it is known that both statements may not hold when the codimension of $M$ is strictly larger than 2 (see [9]).

Nevertheless, $u$ can be taken smooth in the complement of $M \cup S$, where $S$ is an additional singular set of codimension $k+1$ (see Theorem 5.10). Note that we have preferred to state this result for polyhedral chains rather than smooth surfaces, the reason being that polyhedral chains, and not smooth surfaces, are dense in the class of integral currents. Indeed, the original motivation for this research was to provide an upper bound for a $\Gamma$-convergence result for functionals of Ginzburg-Landau type, and this required constructing a map $u$ as above for every $M$ in a suitably dense subclass of integral currents (cf. [1], [2], see also [29] for related lower bound and compactness results).

The paper is organized as follows. In Sect. 2 we set the notation and briefly recall the notions of geometric measure theory which are needed throughout the paper, plus some additional results which are not widely available in reference texts. Section 3 contains the definition of Jacobian and summarize some of the existing results, here included the structure theorem for the Jacobians of $S^{k-1}$-valued maps 
(Theorem 3.8 and Corollary 3.10). In Sects. 4 and 5 we describe two constructions for maps with prescribed singularity, or Jacobian, respectively in the case $k=2$ and $k$ arbitrary (when $k=2$, the special structure of $S^{1}$ allows for a construction which is simpler and more powerful than that for general $k$ ). In Sect. 6 we have gathered some additional remarks on the results described in the previous sections, together with some open problems. For the sake of a clear exposition, we have postponed to Sect. 7 some technical lemmas: a version of the coarea formula for Sobolev maps, the proof of Theorem 3.8, and an approximation result for rectifiable currents.

Acknowledgements. This research was supported by C.N.R. through grant 99.01699.CT01, and by MURST research project "Calcolo delle Variazioni". During a very early stage of the collaboration, the first and third authors were respectively supported by fellowships of the Max Planck Institute for Mathematics in the Sciences (Leipzig) and C.N.R.

\section{Notation and preliminary results}

Throughout this paper $\Omega$ is an open subset of $\mathbb{R}^{n}$ with $n \geq 2 ; S^{k-1}$ is the unit sphere in $\mathbb{R}^{k}$. The constant $\alpha_{k}$ stands for the $k$-dimensional volume of the unit ball in $\mathbb{R}^{k}$; thus $k \alpha_{k}$ is the $(k-1)$-dimensional volume of $S^{k-1}$. The open ball of radius $r$ and center $x$ is denoted by $B(x, r)$, and we simply write $B(r)$ if $x$ is the origin; a $k$-dimensional disk in $\mathbb{R}^{n}$ is an oriented isometric embedding of a closed ball in $\mathbb{R}^{k}$, and is usually denoted by $E$; we may write $E^{k}$ to recall the dimension.

By $k$-surface in $\Omega$ we mean, if not otherwise specified, a smooth, oriented, closed $k$-dimensional submanifold of $\Omega$, possibly with boundary. $\mathcal{H}^{k}$ stands for the $k$-dimensional Hausdorff measure (and agrees with the usual $k$-dimensional volume on every smooth $k$-surface), while $\mathcal{L}^{n}$ is the $n$-dimensional Lebesgue measure; when no doubts may arise, we omit any explicitly mention of the measure in integrals. Sets and maps are always at least Borel measurable.

We write $D u$ to denote indifferently the classical, the approximate, and the distributional derivative (or gradient) of $u$, the precise meaning being usually clear from the context. We denote by $\operatorname{Lip}_{\text {loc }}(\Omega)$ the class of all maps which are locally Lipschitz on $\Omega$. We say that a map is a Sobolev map when it belongs to $W_{\text {loc }}^{1,1}$; we recall that for such maps the distributional and the pointwise (approximate) derivative agree. As usual, $W^{1, p}\left(\Omega, S^{k-1}\right)$ is the class of all $u$ in the Sobolev space $W^{1, p}\left(\Omega, \mathbb{R}^{k}\right)$ which take values in $S^{k-1}$ almost everywhere. For the basic properties of Sobolev functions we refer to [16].

Throughout this paper we will make frequent use of many elementary results and notions from geometric measure theory and differential topology which are not widely available in reference texts. In order to give precise definitions, and allow the reader to verify all formulas, we summarize beforehand the basic notations about forms and currents. We refer the reader to [17], [20], or [35] for detailed expositions of geometric measure theory, and to [25], [22] for differential topology.

2.1. Vectors and covectors. For $k$-vectors and $k$-covectors in $\mathbb{R}^{n}$ we follow the standard notation (see for instance $[35, \S 25]$, or $[20, \S 2.2 .1]$ ) and just recall here 
some basic facts: $\left\{e_{1}, \ldots, e_{n}\right\}$ is the canonical basis of $\mathbb{R}^{n}$, and given a multi-index $\mathbf{i}$ of order $k$, namely $\mathbf{i}:=\left\{i_{1}, \ldots, i_{k}\right\}$ with $1 \leq i_{1}<i_{2}<\cdots<i_{k} \leq n$, we denote by $e_{\mathbf{i}}$ the $k$-vector $e_{i_{1}} \wedge \cdots \wedge e_{i_{k}}$, and by $\widehat{e_{\mathbf{i}}}$ the $(n-k)$-vector associated to the complement of $\mathbf{i}$. The set of all $e_{\mathbf{i}}$ with $\mathbf{i}$ ranging among all multi-indexes of order $k$ is a basis of the space $\wedge_{k}\left(\mathbb{R}^{n}\right)$ of all $k$-vectors in $\mathbb{R}^{n}$. We denote by $d x_{i}$ the 1 -covector (or linear form) which takes $x \in \mathbb{R}^{n}$ into the $i$-th component $x_{i}$, and define the $k$-covector $d x_{\mathbf{i}}$ and the $(n-k)$-covector $\widehat{d x}_{\mathbf{i}}$ as above. The $n$-covector $d x_{1} \wedge \cdots \wedge d x_{n}$ is sometimes simply denoted by $d x$. The set of all $d x_{i}$ with $i=1, \ldots, n$ is the canonical basis of the dual of $\mathbb{R}^{n}$ (the space of 1 -covectors $\wedge^{1}\left(\mathbb{R}^{n}\right)$ ), and the set of all $d x_{\mathbf{i}}$ with $\mathbf{i}$ ranging among all multi-indexes of order $k$ is a basis of the space $\wedge^{k}\left(\mathbb{R}^{n}\right)$ of all $k$-covectors ${ }^{3}$. Vectors and covectors are dual to each other via the duality pairing defined by $d x_{\mathbf{i}} \cdot e_{\mathbf{j}}:=\delta_{\mathbf{i j}}-$ that is, 1 if $\mathbf{i}=\mathbf{j}$, and 0 otherwise.

2.2. Rectifiable sets. A set $M$ is $k$-rectifiable if it has locally finite $\mathcal{H}^{k}$ measure, and can be covered, up to an $\mathcal{H}^{k}$-negligible subset, by a countable family of $k$-dimensional surfaces of class $C^{1}$. For such sets the tangent space $\operatorname{Tan}(M, x)$ is well-defined in a measure theoretic sense at $\mathcal{H}^{k}$-almost every point $x \in M$; an orientation $\tau$ is any Borel map which associates to $x$ a simple unit $k$-vector $\tau(x)$ which spans $\operatorname{Tan}(M, x)$ for $\mathcal{H}^{k}$-a.e. $x \in M$ (cf. [17, §3.2.14-16], or [20, §2.1.4]). If $M$ is an orientable $k$-surface, then the orientation is always taken to be continuous, and induces a canonical orientation on $\partial M$ (so that $\eta \wedge \tau_{\partial M}=\tau_{M}$, where $\eta$ is the unit outer normal to $\partial M$ ).

2.3. Currents and forms. A $k$-form $\omega$ on $\Omega$ is a map (or even a distribution) which takes values in $k$-covectors, and $d \omega$ denotes its (external) differential; the action of a smooth oriented $k$-surface in $\Omega$ on a $k$-form is given by the usual integration (cf. Sect. 2.5).

A $k$-dimensional current on $\Omega$ is a distribution which takes values in $k$-vectors, or equivalently, an element of the dual of the space of smooth $k$-forms with compact support. The action of a current $T$ on a form $\omega$ is sometimes denoted by $T[\omega]$. The boundary of $T$ is defined in order to satisfy Stokes theorem, that is, $\partial T[\omega]:=T[d \omega]$ for every $k$-form $\omega$ of class $C_{c}^{\infty}$.

The mass $\|T\|$ of $T$ is its total variation (as a distribution); therefore $T$ has (locally) finite mass if and only if it can be represented as a (locally) bounded measure valued in $k$-vectors, that is, as the product of a (locally) bounded positive measure $\mu$ and an orientation $\tau$, namely a $\wedge_{k}\left(\mathbb{R}^{n}\right)$-valued map such that $|\tau|=1$ $\mu$-a.e. The measure $\mu$ is called variation of $T$, and denoted by $|T|$.

2.4. Basic operations on currents and forms. Given a proper map $f: \Omega \rightarrow \Omega^{\prime}$, the pull-back of $k$-form $\omega$ on $\Omega^{\prime}$ according to $f$ is the $k$-form on $\Omega$ defined by

3 We endow $\wedge_{k}\left(\mathbb{R}^{n}\right)$ and $\wedge^{k}\left(\mathbb{R}^{n}\right)$ with the euclidean norms associated to the previous basis. These are different from the usual mass and co-mass, but sometimes more convenient. On the other hand, the euclidean norm and the mass agree for simple vectors (those which can be written as a wedge product of 1-vectors), and therefore the difference is not relevant to most of this paper. 
$f^{\sharp} \omega(x):=(D f(x))^{\sharp} \omega(f(x)),{ }^{4}$ while the push-forward $f_{\sharp} T$ of a $k$-current $T$ on $\Omega$ is the $k$-current on $\Omega^{\prime}$ defined by the obvious duality (that is, $f_{\sharp}$ is the adjoint of $\left.f^{\sharp}\right)$. Recall that $d\left(f^{\sharp} \omega\right)=f^{\sharp}(d \omega)$ and $\partial\left(f_{\sharp} T\right)=f_{\sharp}(\partial T)$.

The cartesian product of a $k_{1}$-current $T_{1}$ in $\mathbb{R}^{n}$ and a $k_{2}$-current $T_{2}$ in $\mathbb{R}^{m}$ is a $\left(k_{1}+k_{2}\right)$-current $T_{1} \times T_{2}$ in $\mathbb{R}^{n} \times \mathbb{R}^{m}$. In particular, if $T_{1}=\tau_{1} \cdot \mu_{1}$ and $T_{2}=\tau_{2} \cdot \mu_{2}$, then $T_{1} \times T_{2}=\tau \cdot \mu$ where $\mu$ is the product measure $\mu_{1} \times \mu_{2}$, and $\tau(x, y):=\tau_{1}(x) \wedge \tau_{2}(y) .^{5}$

2.5. Integral and polyhedral currents. Given a rectifiable set $M$ equipped with an orientation $\tau_{M}$ and a multiplicity $\sigma_{M}$ (namely a real function which is locally summable on $M$ ), we can define a current $T$ by

$$
T[\omega]:=\int_{M} \sigma_{M}\left(\omega \cdot \tau_{M}\right) d \mathcal{H}^{k} \quad \text { for every } k \text {-form } \omega \text { of class } C_{c}^{\infty} .
$$

In other words, $T$ is the $k$-vector-valued measure given by $\sigma_{M} \cdot \tau_{M} \cdot \mathcal{H}^{k}\llcorner M$ (the last term stands for the restriction of the measure $\mathcal{H}^{k}$ to the set $M$ ). Thus the variation of $T$ is the positive measure $|T|=\left|\sigma_{M}\right| \cdot \mathcal{H}^{k}\llcorner M$, and the mass is $\|T\|=\int_{M}\left|\sigma_{M}\right| d \mathcal{H}^{k}$, that is, the measure of the set $M$ counted with multiplicity.

We may occasionally denote this current simply by $M$.

When $M$ is a regular $k$-surface, the current canonically associated to $M$ is given by formula (2.1) with the multiplicity $\sigma_{M}$ set equal to 1 everywhere.

A current $T$ is called rectifiable if it can be represented as in (2.1) with an integer multiplicity $\sigma_{M}$, and integral if both $T$ and $\partial T$ are rectifiable. A polyhedral current in $\mathbb{R}^{n}$ is a finite sum of $k$-dimensional oriented simplexes $S_{i}$ endowed with constant integer multiplicities $\sigma_{i}$. It is also usually assumed that $S_{i} \cap S_{j}$ is either empty or a common face (of any dimension) of $S_{i}$ and $S_{j}$.

Note that polyhedral currents are just the usual simplicial chains of algebraic topology, and the boundary coincides with the usual algebraic object; they are particularly relevant because they are dense in the class of integral currents (with respect to flat convergence with convergence of masses).

Remark 2.6. Every integral $k$-current $M$ in $\mathbb{R}^{n}$ with finite mass, bounded support, and no boundary is the boundary of an integral current $N$ with finite mass, e.g., the cone over $M$ with vertex at the origin (more precisely, the push-forward of the product current $M \times(0,1)$ in $\mathbb{R}^{n} \times \mathbb{R}$ according to the map $\left.(x, t) \mapsto t x\right)$. Then when $M$ is a polyhedral current, $N$ is polyhedral, too.

2.7. Further operations on vectors and covectors. There is a canonical way to identify $k$-covectors and $(n-k)$-vectors, namely the operator $\star$ which takes every

\footnotetext{
${ }^{4}$ Given a linear map $L: \mathbb{R}^{n} \rightarrow \mathbb{R}^{m}$, then $L^{\sharp}: \wedge^{k}\left(\mathbb{R}^{m}\right) \rightarrow \wedge^{k}\left(\mathbb{R}^{n}\right)$ is defined by $L^{\sharp}\left(\beta_{1} \wedge \cdots \wedge \beta_{k}\right):=\beta_{1} L \wedge \cdots \wedge \beta_{k} L$ for simple $k$-covectors, and then extended by linearity.

5 Here one tacitly identifies multi-vectors in $\mathbb{R}^{n}$ and $\mathbb{R}^{m}$ with those in $\mathbb{R}^{n} \times\{0\}$ and $\{0\} \times \mathbb{R}^{m}$, respectively.
} 
$k$-covector $\beta$ into the $(n-k)$-vector $\star \beta$ defined by the identity ${ }^{6}$

$$
\alpha \cdot \star \beta=(\alpha \wedge \beta) \cdot\left(e_{1} \wedge \cdots \wedge e_{n}\right) \quad \text { for every } \alpha \in \wedge^{n-k}\left(\mathbb{R}^{n}\right) .
$$

Thus $\star$ takes $k$-forms into $(n-k)$-currents; moreover the exterior derivative is conjugate to the boundary operator via $\star$, in the sense that

$$
\star(d \omega)=(-1)^{n-k} \partial(\star \omega) .
$$

If we apply an $h$-covector $\beta$ to a $k$ vector $v$ with $k<h$, we get an $(h-k)$-covector, denoted by $\beta \mathrm{L} v$, which is defined by the identity ${ }^{7}$

$$
\left(\beta\llcorner v) \cdot w=\beta \cdot(v \wedge w) \text { for every } w \in \wedge_{h-k}\left(\mathbb{R}^{n}\right) .\right.
$$

One easily checks that

$$
\star(\beta\llcorner v)=(\star \beta) \wedge v .
$$

2.8. Convolution of currents and forms. The convolution of two maps $U: \mathbb{R}^{n} \rightarrow$ $\wedge_{h}\left(\mathbb{R}^{n}\right)$ and $T: \mathbb{R}^{n} \rightarrow \wedge_{k}\left(\mathbb{R}^{n}\right)$ (resp. an $h$ - and a $k$-current) is defined by

$$
U * T(x):=\int_{\mathbb{R}^{n}} U(x-y) \wedge T(y) d y .
$$

Since $|U * T| \leq|U| *|T|$, all the usual inequalities for the convolution of real functions hold in this case, too. For instance, $U * T$ is a well-defined map in, say, $L^{1}$ if both $U$ and $T$ belong to $L^{1}$, and $\|U * T\|_{1} \leq\|U\|_{1}\|T\|_{1}$. Moreover definition (2.4) can be extended, as for the usual convolution, to the case both $T$ and $U$ are bounded measures, or to the case $T$ is a distribution and $U$ is of class $C_{c}^{\infty}$.

Since $U * T$ is the push-forward of the product current $U \times T$ according to the map $\Phi: \mathbb{R}^{n} \times \mathbb{R}^{n} \rightarrow \mathbb{R}^{n}$ which takes $(x, y)$ into $x+y$, the well-known formula $\partial(U \times T)=\partial U \times T+(-1)^{h} U \times \partial T$ gives

$$
\partial(U * T)=\partial U * T+(-1)^{h} U * \partial T,
$$

provided all convolution products in (2.5) are well-defined (at least as distributions).

The convolution of an $h$-form $\omega: \mathbb{R}^{n} \rightarrow \wedge^{h}\left(\mathbb{R}^{n}\right)$ and a $k$-current $T: \mathbb{R}^{n} \rightarrow$ $\wedge_{k}\left(\mathbb{R}^{n}\right)$ is defined by the obvious analogous of (2.4) - with the $\wedge$-product replaced by the L-product - and enjoys the same basic properties. In particular, identity (2.3) yields $\star(\omega * T)=(\star \omega) * T$, and then (2.5) implies

$$
d(\omega * T)=(-1)^{k}[d \omega * T+\omega * \partial T] .
$$

6 A direct definition is $\star d x_{\mathbf{i}}:=\sigma\left(\mathbf{i}^{\prime}, \mathbf{i}\right) \widehat{e_{\mathbf{i}}}$ for every multi-index $\mathbf{i}$ of order $k$, where $\mathbf{i}^{\prime}$ is the set of indices not contained in $\mathbf{i}$ and $\sigma\left(\mathbf{i}^{\prime}, \mathbf{i}\right)$ is the sign of the permutation $\left(\mathbf{i}^{\prime}, \mathbf{i}\right)$. Up to the identification of $k$-vectors and $k$-covectors induced by the scalar product, $\star$ is the usual Hodge operator.

7 A direct definition is the following: given multi-indexes $\mathbf{i}$ and $\mathbf{j}$ of order $h$ and $k$ respectively, then $d x_{\mathbf{i}} \mathbf{L} e_{\mathbf{j}}:=0$ if $\mathbf{j} \not \subset \mathbf{i}$, and $d x_{\mathbf{i}} \mathbf{L} e_{\mathbf{j}}:=\sigma\left(\mathbf{j}, \mathbf{i}^{\prime}\right) d x_{\mathbf{i}^{\prime}}$ otherwise, where $\mathbf{i}^{\prime}:=\mathbf{i} \backslash \mathbf{j}$ is the set of indices in $\mathbf{i}$ not contained in $\mathbf{j}$. Up to a sign, this is the "elbow" interior multiplication of $[17, \S 1.5 .1]$. 
2.9. Forms on manifolds and submanifolds. An $h$-form on a smooth $k$-dimensional manifold $M$ is a map which associates to every $y \in M$ an $h$-covector on $\operatorname{Tan}(M, y){ }^{8}$ The restriction of an $h$-covector $\alpha$ in $\mathbb{R}^{n}$ to a $k$-dimensional subspace $T$ of $\mathbb{R}^{k}$ is the $h$-covector on $T$ which represents the restriction of the dual map $v \mapsto \alpha \cdot v$ to $h$-vectors $v$ in $\wedge_{h}(T)$. Accordingly, if $M$ is a $k$-surface in $\mathbb{R}^{n}$, the restriction of an $h$-form $\omega$ on $\mathbb{R}^{n}$ to $M$ is the map which takes every $y \in M$ into the restriction of $\omega(y)$ to $\operatorname{Tan}(M, y)$; we say that the $h$-form $\omega$ on $\mathbb{R}^{n}$ extends the $h$-form $\omega^{\prime}$ on $M$ if $\omega^{\prime}$ agrees with the restriction of $\omega$ to $M$. For forms of class $C^{1}$ the exterior differential commutes with the restriction, that is, $d \omega^{\prime}$ is the restriction of $d \omega$.

A particularly relevant $(k-1)$-form on $\mathbb{R}^{k}$ is

$$
\omega_{0}(y):=\sum_{i=1}^{k}(-1)^{i-1} y_{i} \widehat{d y_{i}} .
$$

Indeed $d \omega_{0}=k d y_{1} \wedge \cdots \wedge d y_{k}=k d y$, while the restriction of $\omega_{0}$ to the unit sphere $S^{k-1}$ is the standard volume form on $S^{k-1}$.

2.10. Brouwer degree and area formula. Given $k$-dimensional oriented manifolds $M$ and $M^{\prime}, M$ compact and $M^{\prime}$ without boundary, and a continuous map $f$ : $M \rightarrow M^{\prime}$, then $\operatorname{deg}\left(f, M, M^{\prime}, y\right)$ denotes the Brouwer degree of $f$ at the point $y \in M^{\prime} \backslash f(M)$ (see [25, Chap. 5], and [22, Chap. 3]).

If $f$ is of class $C^{1}$ and $y$ is a regular value of $f$, then $\operatorname{deg}\left(f, M, M^{\prime}, y\right)$ is the number of points $x \in f^{-1}(y)$ where the derivative $D f(x)$ is orientationpreserving, minus the number of those where the derivative is orientation-reversing. We recall that the degree is constant on each connected component of $M^{\prime} \backslash f(\partial M)$; in particular, if $\partial M=\varnothing, \operatorname{deg}\left(f, M, M^{\prime}, y\right)$ does not depend on $y$, and we simply write $\operatorname{deg}\left(f, M, M^{\prime}\right)$, or even $\operatorname{deg}(f)$.

Using the degree we can write an "oriented" version of the area formula: for every $k$-form $\omega$ on $M^{\prime}$

$$
\int_{M}\left(f^{\sharp} \omega\right) \cdot \tau_{M} d \mathcal{H}^{k}=\int_{M^{\prime}} \operatorname{deg}(f, M, \cdot) \omega \cdot \tau_{M^{\prime}} d \mathcal{H}^{k} .
$$

The following fact will be needed later: if $M^{\prime}=\mathbb{R}^{k}$, and $f$ maps $\partial M$ into $S^{k-1}$, then $\operatorname{deg}\left(u, M, \mathbb{R}^{k}, y\right)=0$ for $|y|>1$ and

$$
\operatorname{deg}\left(u, M, \mathbb{R}^{k}, y\right)=\operatorname{deg}\left(f, \partial M, S^{k-1}\right) \quad \text { for }|y|<1 .
$$

Indeed $\operatorname{deg}(u, M, \cdot)$ is constant on both components of $\mathbb{R}^{k} \backslash S^{k-1}$, and must vanish in the unbounded one. Moreover, denoting by $d$ its value on the unit ball of $\mathbb{R}^{k}$ and taking $\omega_{0}$ as in (2.7),

$$
\begin{aligned}
\alpha_{k} d=\int_{\mathbb{R}^{k}} \operatorname{deg}\left(f, M, \mathbb{R}^{k}, y\right) d y & =\int_{M} f^{\sharp}(d y) \cdot \tau_{M} d \mathcal{H}^{k} \\
& =\int_{M} \frac{1}{k} d f^{\sharp}\left(\omega_{0}\right) \cdot \tau_{M} d \mathcal{H}^{k} \\
& =\int_{\partial M} \frac{1}{k} f^{\sharp}\left(\omega_{0}\right) \cdot \tau_{\partial M} d \mathcal{H}^{k-1}=\alpha_{k} \cdot \operatorname{deg}(f, \partial M),
\end{aligned}
$$

\footnotetext{
8 To be precise, this is a section of the $h$-cotangent bundle of $M$.
} 
where the second and fifth equalities follow from the area formula, the third one from the identity $d y=\frac{1}{k} d \omega_{0}$, and the fourth one from Stokes theorem.

2.11. Intersection and linking numbers. Let $M$ and $M^{\prime}$ be surfaces in $\mathbb{R}^{n}$ with dimensions $n-k$ and $k$, respectively, endowed with (locally constant) multiplicities $\sigma_{M}$ and $\sigma_{M^{\prime}}$. If $M$ and $M^{\prime}$ are transversal and $\partial M \cap M^{\prime}=M \cap \partial M^{\prime}=\varnothing$, then the intersection number of $M$ and $M^{\prime}, \operatorname{int}\left(M, M^{\prime}\right)$, is defined as the sum over all $x \in M \cap M^{\prime}$ of $\pm \sigma_{M}(x) \cdot \sigma_{M^{\prime}}(x)$, where the sign is + if $\tau_{M}(x) \wedge \tau_{M^{\prime}}(x)$ agrees with $e_{1} \wedge \cdots \wedge e_{n}$, and is - if the opposite holds (cf. [25, Sect. 5.2], or [22, §3.5]).

If $M^{\prime \prime}$ is a $(k-1)$-surface in $\mathbb{R}^{n}$ endowed with multiplicity $\sigma_{M^{\prime \prime}}$, and $\partial M, \partial M^{\prime \prime}$ and $M \cap M^{\prime \prime}$ are all empty, the linking number of $M$ and $M^{\prime \prime}, \operatorname{link}\left(M, M^{\prime \prime}\right)$, is defined as the degree - computed taking the multiplicities into account - of the map that takes $(x, y) \in M \times M^{\prime \prime}$ into $(x-y) /|x-y| \in S^{n-1}$ (cf. [25, Sect. 5.1, Exercise 7]).

The relation between intersection and linking number is given by the following formula: if $M$ and $M^{\prime}$ are as above, and $M$ has no boundary, then

$$
\operatorname{int}\left(M, M^{\prime}\right)=(-1)^{k} \operatorname{link}\left(M, \partial M^{\prime}\right) .
$$

Take indeed $g: M \times M^{\prime} \rightarrow[0,1]$ such that $g(x, y)=1$ when $(x, y) \in M \times \partial M^{\prime}$, $g(x, y)>0$ unless $x=y$, and $g(x, y)=|x-y|$ for $|x-y|$ sufficiently small. Set $\Phi(x, y):=g(x, y) \cdot(x-y) /|x-y|$ for all $(x, y) \in M \times M^{\prime}$. Then $\operatorname{link}\left(M, \partial M^{\prime}\right)$ is equal to $\operatorname{deg}\left(\Phi, M \times \partial M^{\prime}, S^{k-1}\right)$ which is equal to $\operatorname{deg}\left(\Phi, M \times \partial M^{\prime}, \mathbb{R}^{k}, 0\right)$ by (2.8), and it is easily verified that the latter is $(-1)^{k} \operatorname{int}\left(M, M^{\prime}\right)$.

\section{Jacobians of Sobolev maps}

In this section we recall the basic definitions and main results about the Jacobian of Sobolev maps (see [28] for further details).

3.1. Differentials and Jacobians. The differential of a scalar function $u$ on $\Omega$ is, as usual, the 1-form $d u:=\sum_{1}^{n} D_{i} u d x_{i}$, where $D_{i}$ denotes the $i$-th partial derivative. For $k \leq n$, the $k$-dimensional Jacobian of a (smooth) map $u=\left(u_{1}, \ldots, u_{k}\right)$ : $\mathbb{R}^{n} \rightarrow \mathbb{R}^{k}$ is the pull-back according to $u$ of the volume form $d y$ on $\mathbb{R}^{k}$, that is

$$
J u:=u^{\sharp}(d y)=d u_{1} \wedge \cdots \wedge d u_{k},
$$

and since $d y=\frac{1}{k} d \omega_{0}$, where $\omega_{0}$ is given in (2.7), then

$$
J u=\frac{1}{k} d u^{\sharp}\left(\omega_{0}\right)=\frac{1}{k} d\left(\sum_{i=1}^{k}(-1)^{i-1} u_{i} \widehat{d u_{i}}\right) .
$$

While (3.1) makes sense for maps $u$ with gradient in $L^{k},(3.2)$ makes sense (as a distribution) as long as the products within brackets are well-defined, e.g. for bounded maps $u$ with gradient in $L^{k-1}$. This motivates the following definition (cf. [28]): 
Definition 3.2. The Jacobian of a map $u: \Omega \rightarrow \mathbb{R}^{k}$ of class $L_{\mathrm{loc}}^{\infty} \cap W_{\mathrm{loc}}^{1, k-1}$ is the $k$-form with distributional coefficient Ju defined in (3.2).

Remark 3.3. For many purposes it is sometimes more convenient to consider, instead of the $k$-form $J u$, the $(n-k)$-current $\star J u$, where $\star$ is the operator defined in Sect. 2.7. Since $J u$ is an exterior differential (cf. (3.2)), then $\star J u$ is always a boundary, and in particular $\partial(\star J u)=0$.

Remark 3.4. The Jacobian operator $J$, although nonlinear, is continuous, in the sense that given any bounded closed set $E \subset \mathbb{R}^{k}$ and a sequence of maps $u_{j} \in W^{1, k-1}(\Omega, E)$ which converge strongly to $u$, then the Jacobians $J u_{j}$ converge to $J u$ in the sense of distributions: in fact, the expression within brackets at the right-hand side of (3.2) converges strongly in the $L^{1}$-norm. ${ }^{9}$ It follows that (3.1) and (3.2) agree for all maps of class $W_{\mathrm{loc}}^{1, k}$, and in particular

$$
J u=\sum \operatorname{det}\left(D_{\mathbf{i}} u\right) d x_{\mathbf{i}},
$$

where the sum is taken over all multi-indexes $\mathbf{i}$ of order $k$, and $D_{\mathbf{i}} u$ denotes the $k \times k$ matrix with columns $D_{i_{1}} u, \ldots, D_{i_{k}} u$.

Remark 3.5. Assuming that $u$ is sufficiently regular (say, of class $W^{1, k}$ ), the notion of Jacobian is better understood in some special cases:

o for $k=1, J u$ is just the differential of $u$;

$\circ$ for $k=2$ and $n=3, \star J u$ is the vector product $D u_{1} \times D u_{2}$;

o for $k=n, J u=\operatorname{det}(D u) d x$ and $\star J u=\operatorname{det}(D u)$.

If $k=n$ and $u$ is a map of class $L^{\infty} \cap W^{1, n-1}$, then $\star J u$ is the distributional determinant introduced in [5], and usually denoted by $\operatorname{Det}(D u)$. Although $\operatorname{Det}(D u)$ may not agree with the pointwise determinant $\operatorname{det}(D u)$ for maps in $W^{1, p}$ with $p<k$, it was proved in [31] that if $\operatorname{Det}(D u)$ is a measure, then its absolutely continuous part (with respect to Lebesgue measure) is represented by $\operatorname{det}(D u)$. Using this result and a standard slicing technique (cf. [28, Sect. 5]), it is easy to show that if $J u$ has bounded mass then its absolutely continuous part is represented by the $k$-form at the right-hand side of (3.1) or (3.3).

\section{$S^{k-1}$-valued maps}

For the rest of this paper we confine our attention to maps with values in the sphere $S^{k-1}$. First of all we remark that for any $u \in W_{\text {loc }}^{1, k}\left(\Omega, S^{k-1}\right)$ the Jacobian $J u$ is just pointwise defined as the pull-back of the standard volume form on $\mathbb{R}^{k}$ (cf. Remark 3.4) and therefore is 0 , because $\mathcal{L}^{k}\left(S^{k-1}\right)=0$.

The situation is different if we consider maps $u \in W^{1, k-1}\left(\Omega, S^{k-1}\right)$. For simplicity, let us first assume that $u$ is regular (say, locally Lipschitz) in the complement

9 This result still holds if we replace strong convergence in $W^{1, k-1}$ by weak convergence in $W_{\text {loc }}^{1, k+\varepsilon}$ for some $\varepsilon>0$, and even $\varepsilon=0$ when $k=2$. This stronger statement can be proved by induction on $k$. 
of a closed singular set $S$. The previous argument shows that the restriction of the distribution $J u$ to $\Omega \backslash S$ vanishes, that is, $J u$ is supported on $S$. If in addition the singular set $S$ is a regular surface of codimension $k$, then $J u$ can be expressed in terms of $S$ and the degree of the singularity of $u$ at $S$, as shown in the paragraphs below.

3.6. Maps with regular singularities, case $n=k$. When $S=\left\{x_{i}\right\}$ is a finite set, it is not difficult to show that $\star J u$ is a linear combination of Dirac masses centered at the points $x_{i}$. Moreover, if we take for every $i$ a ball $B_{i}$ which contains $x_{i}$ and no other point of $S$, and set $d_{i}:=\operatorname{deg}\left(u, \partial B_{i}, S^{k-1}\right)$, then

$$
\star J u=\alpha_{k} \sum d_{i} \cdot \delta_{x_{i}} .
$$

This formula can be found in [14] (see also [28, Example 3 of Sect. 3]). A sketch of proof is the following: knowing that $\star J u$ is of the form $\sum \beta_{i} \delta_{x_{i}}$, and denoting by $\tau_{B_{i}}=e_{1} \wedge \cdots \wedge e_{k}$ the standard orientation of $B_{i}$, then

$$
\beta_{i}=\int_{B_{i}} J u \cdot \tau_{B_{i}}=\int_{\partial B_{i}} \frac{1}{k} u^{\sharp}\left(\omega_{0}\right) \cdot \tau_{\partial B_{i}}=\frac{1}{k} \mathcal{H}^{k-1}\left(S^{k-1}\right) d_{i}=\alpha_{k} d_{i},
$$

where the second equality follows from Stokes theorem and definition (3.2), and the third one from the area formula and the fact that $\omega_{0}$ is the volume form on $S^{k-1}$.

3.7. Maps with regular singularities, case $n>k$. If $u$ belongs to $W^{1, k-1}\left(\Omega, S^{k-1}\right)$ and is regular outside a Lipschitz surface $S$ with codimension $k$ and no boundary in $\Omega$, then (cf. [28, Example 4 of Sect. 3])

$$
\star J u=\alpha_{k} \cdot M,
$$

where $M$ is the integral current associated to $S$ and to the multiplicity $\sigma$ defined for every $x \in S$ by the formula

$$
\sigma(x)=\operatorname{deg}\left(u, \partial E, S^{k-1}\right),
$$

where $E$ is a $k$-dimensional disk in $\Omega$ which satisfies $S \cap E_{x}=\{x\}$, is transversal to $S$, and oriented so that $\tau_{S}(x) \wedge \tau_{E}(x)=e_{1} \wedge \cdots \wedge e_{n}$.

Note that $\star J u$ has no boundary and is supported on a regular surface $S$ with same dimension and no boundary, and therefore $\sigma$ must be constant on every connected component of $S$ (e.g., by the constancy theorem - see [20, §2.2.3]). Formula (3.5) can be derived from (3.4) using a dimension reduction argument based on slicing formulas for the Jacobian (see [28, Sect. 5]).

We can generalize formula (3.5) using the notions introduced in \$2.11: for every $k$-surface $A$ relatively compact in $\Omega$ which is transversal to $S$ and such that $\partial A \cap S=\varnothing$, the intersection number of $S$, endowed with multiplicity $\sigma$, and $A$ is equal to $\operatorname{deg}\left(u, \partial A, S^{k-1}\right)$. Moreover, if $\Omega=\mathbb{R}^{n}$, formula (2.9) yields

$$
\operatorname{int}(S, A)=(-1)^{k} \operatorname{link}(S, \partial A)=\operatorname{deg}\left(u, \partial A, S^{k-1}\right) .
$$




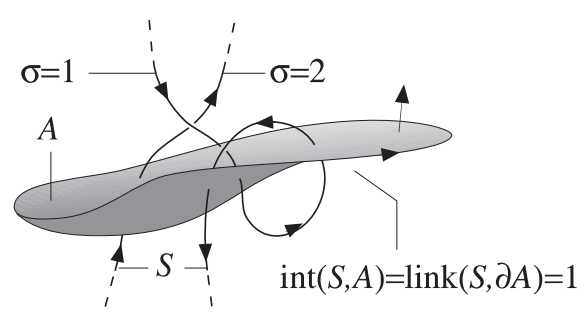

Fig. 1.

Identity (3.6) completely characterizes the multiplicity $\sigma$, and therefore also $\star J u$ (see Fig. 1 for an example with $n=3, k=2$ ).

Paragraphs 3.6 and 3.7 show that the current $\star J u$ is related to the part of the singular set of $u$ which is topologically meaningful. In fact, the same conclusions holds also for maps in $W^{1, k-1}$ which are continuous in the complement of a finite sum of Lipschitz surfaces with codimension $k$ and no boundary (a discrete set in the case $n=k$ ).

Of course, not all maps in $W^{1, k-1}$ are so regular. Yet, for $n=k$, the Jacobian $J u$ can still be represented as in (3.4) for suitable $x_{i} \in \Omega$ and $d_{i} \in \mathbb{Z}$ (cf. [28, Proposition 1 of Sect. 5]), although in this case it is more difficult to interpret the integers $d_{i}$ as degrees and the points $x_{i}$ as singularities. In the general case we have the following structure theorem (cf. [23] for maps of class $W^{1-1 / k, k}$ ):

Theorem 3.8. If $u$ belongs to $W^{1, k-1}\left(\Omega, S^{k-1}\right)$, then $\star J u$ can be represented as $\star J u=\alpha_{k} \partial N$, where $N$ is a rectifiable current with codimension $k-1$ in $\Omega$. More precisely, we can take $N=(-1)^{n-k+1} N_{y}$ where $N_{y}$ is any regular level set of $u$ (in the sense of Sect. 7.5), and we can choose y so that

$$
\|N\| \leq \frac{1}{k \alpha_{k}} \int_{\Omega}|D u|^{k-1} .
$$

Corollary 3.9. If $u \in W^{1, k-1}\left(\Omega, S^{k-1}\right)$ and $\phi$ is a Lipschitz map of $S^{k-1}$ into itself, then the Jacobian of the composition $\phi \circ u$ is $J(\phi \circ u)=\operatorname{deg}(\phi) J u$.

Corollary 3.10 (see [27]). If $u$ belongs to $W^{1, k-1}\left(\Omega, S^{k-1}\right)$ and Ju has locally finite mass, then

$$
\star J u=\alpha_{k} M
$$

where $M$ is an integral current in $\Omega$ with codimension $k$ and no boundary.

The proof of Theorem 3.8 has been postponed to Sect. 7. For smooth $\phi$, Corollary 3.9 follows immediately by the characterization of $\star J u$ as boundary of any regular level set of $u$ and the definition of degree, while the general case is obtained by approximation. Corollary 3.10 is a straightforward consequence of Theorem 3.8 and the boundary rectifiability theorem of Federer and Fleming (see [35, Theorem 30.3], or [17, Theorem 4.2.16(2)]). 
In [28] the rectifiability of Jacobian is proved by a dimension-reduction argument based on a rectifiability criterion by slicing proved in [37], [26]. Alternatively, one can use the product structure of the boundary of graphs with finite mass ([21, \$4.2.1]) and, again, the boundary rectifiability theorem (cf. Remark 7.10).

\section{$S^{1}$-valued maps}

In this subsection we fix some notation for the case $k=2$. If we identify $\mathbb{R}^{2}$ with the complex field, the map $\theta \mapsto \exp (2 \pi i \theta)$ is an isometry (up to a scaling factor $2 \pi$ ) of the quotient space $\mathbb{R} / \mathbb{Z}$ into $S^{1}$. Therefore for every map $u: \Omega \rightarrow S^{1}$ there exists exactly one map $\theta: \Omega \rightarrow \mathbb{R} / \mathbb{Z}$ such that $u=\exp (2 \pi i \theta)$.

Furthermore, for every $C^{1}$ function $\theta, d \theta$ is a well-defined 1 -form ${ }^{10}$, and

$$
2 \pi d \theta=u_{1} d u_{2}-u_{2} d u_{1}=-i \bar{u} d u \text { and } 2 \pi|d \theta|=|D u| .
$$

Based on these identities, we say that $\theta$ is of class $W^{1, p}$ when $u$ is $\operatorname{so}^{11}$. However, since $\theta$ is not a real-valued function, $d(d \theta)$ may not vanish, and indeed the first identity in (3.8) implies

$$
\pi d(d \theta)=\frac{1}{2} d\left(u_{1} d u_{2}-u_{2} d u_{1}\right)=\frac{-i}{2} d(\bar{u} d u)=J u .
$$

3.11. Lifting Sobolev maps. When can we lift a $\operatorname{map} \theta: \Omega \rightarrow \mathbb{R} / \mathbb{Z}$ to a map with values in $\mathbb{R}$ ? If we disregard regularity, this is clearly always possible. On the other hand, if $\theta$ is of class $W^{1,1}$ and we look for a lifting with the same regularity, then it must be $d(d \theta)=0$.

If $\Omega$ is simply-connected, this condition is also sufficient (and the lifting is uniquely determined up to integer constants). Hence a map $u \in W^{1,1}\left(\Omega, S^{1}\right)$ can be written as $u=\exp (2 \pi i \theta)$ for some $\theta \in W^{1,1}(\Omega, \mathbb{R})$ if and only if $J u=0$ as a distribution (cf. [15], see also [8], [11] for more general results). In fact, this is an immediate consequence of the following classical lemma on exact forms/conservative vectorfields: if $\Omega$ is simply connected, a 1-form $\omega \in L^{1}(\Omega)$ is the differential of a real-valued function $\theta \in W^{1,1}(\Omega)$ if and only if $d \omega=0$ (as a distribution).

\section{Maps with prescribed singularity: construction for $k=2$}

In this section we address the converse of Theorem 3.8 in codimension two, that is, we construct an $S^{1}$-valued map $u$ with prescribed Jacobians $M$ (Theorem 4.4). For the rest of this section we assume that $\Omega=\mathbb{R}^{n}$. We begin with an elementary geometric construction.

\footnotetext{
10 The tangent space to $\mathbb{R} / \mathbb{Z}$ at every point is identified with $\mathbb{R}$.

11 Since the distributional derivative of a Sobolev function agrees with the approximate derivative, $d \theta$ is still a well-defined 1-form of class $L^{p}$; in other words, the complex 1-form $-i \bar{u} d u$ has no imaginary part. This is no longer true for $B V$ functions; the point is that the distributional derivative of a $\mathbb{R} / \mathbb{Z}$-valued function cannot be defined independently of the embedding of $\mathbb{R} / \mathbb{Z}$ in some euclidean space.
} 
4.1. Geometric construction. Let $M$ be an oriented, boundaryless smooth surface with codimension two (and multiplicity 1 ) in $\mathbb{R}^{n}$. As explained in Sect. 3.7, a map $u: \mathbb{R}^{n} \rightarrow S^{1}$ which is smooth in the complement of $M$ satisfies $\star J u=\pi M$ if (and only if)

$$
\operatorname{deg}\left(u, \gamma, S^{1}\right)=\operatorname{link}(M, \gamma) \quad \text { for every closed curve } \gamma \text { in } \mathbb{R}^{n} \backslash M .
$$

If we write $u=\exp (2 \pi i \theta)$ with $\theta: \mathbb{R}^{n} \backslash M \rightarrow \mathbb{R} / \mathbb{Z}$ smooth, and denote by $\omega_{M}$ the differential of $\theta$, condition (4.1) can be re-written in terms of $\omega_{M}$ as

$$
\int_{\gamma} \omega_{M} \cdot \tau_{\gamma}=\operatorname{link}(M, \gamma) \text { for every closed curve } \gamma \text { in } \mathbb{R}^{n} \backslash M \text {. }
$$

Let now be given a smooth 1 -form $\omega_{M}$ on $\mathbb{R}^{n} \backslash M$ which satisfies (4.2). Then the integral of $\omega_{M}$ on every closed curve in $\mathbb{R}^{n} \backslash M$ is an integer, which implies that $\omega_{M}$ is the differential of a smooth map $\theta: \mathbb{R}^{n} \backslash M \rightarrow \mathbb{R} / \mathbb{Z},{ }^{12}$ and the map $u:=\exp (2 \pi i \theta)$ satisfies $\star J u=\pi M$.

It remains to construct such a form $\omega_{M}$. Recall that $\operatorname{link}(M, \gamma)=\operatorname{deg}(\Phi, M \times \gamma$, $\left.S^{n-1}\right)$, where $\Phi(x, y):=(x-y) /|x-y|$ for every $x \neq y \in \mathbb{R}^{n}$. On the other hand, denoting by $\tilde{\omega}$ the pull-back of the volume form on $S^{n-1}$ according to $\Phi$, by the area formula $n \alpha_{n} \operatorname{deg}\left(\Phi, M \times \gamma, S^{n-1}\right)$ is the integral of $\tilde{\omega}$ on $M \times \gamma$, and then (cf. Sect. 2.7)

$$
\begin{aligned}
n \alpha_{n} \operatorname{link}(M, \gamma) & =\int_{M \times \gamma} \tilde{\omega}(x, y) \cdot\left(\tau_{M}(x) \wedge \tau_{\gamma}(y)\right) \\
& =\int_{\gamma}\left[\int_{M} \tilde{\omega}(x, y) \mathbf{L} \tau_{M}(x)\right] \cdot \tau_{\gamma}(y) .
\end{aligned}
$$

Hence the 1-form defined by the integral within square brackets in (4.3) satisfies (4.2) up to a factor $n \alpha_{n}$, and therefore we just set

$$
\omega_{M}(y):=\frac{1}{n \alpha_{n}} \int_{M} \tilde{\omega}(x, y)\left\llcorner\tau_{M}(x) \text { for every } y \in \mathbb{R}^{n} \backslash M .\right.
$$

When the surface $M$ is replaced by a less regular surface, or by a rectifiable current, the construction described above must be suitably re-interpreted. Notice that the map $\Phi(x, y)$ used to define $\tilde{\omega}(x, y)$ in Sect. 4.1 depends only on $x-y$, and this suggests that the right-hand side of (4.4), and ultimately also $\theta$, can be re-written as convolution products (in the sense of Sect. 2.8). We show in Sect. 4.3 below that this is indeed the case. To this purpose, we first recall some basic facts about $B V$ functions.

12 A continuous 1 -form $\omega$ on a connected open set $\Omega \subset \mathbb{R}^{n}$ is the differential of a map $\theta: \Omega \rightarrow \mathbb{R} / \mathbb{Z}$ of class $C^{1}$ if and only if the integral of $\omega$ along any closed curve in $\Omega$ is an integer. To construct such a $\theta$ it suffices to fix a base-point $x_{0} \in \Omega$, and set, for every $x \in \Omega$, $\theta(x):=\int_{\gamma} \omega \cdot \tau_{\gamma}$, where $\gamma$ is any path connecting $x_{0}$ and $x$. 
4.2. Differentials of $B V$ functions. The measure which represents the distributional differential of a real-valued $B V$ function $v$ can be uniquely decomposed as (see [3, Sect. 3.9])

$$
d v=d_{\mathrm{a}} v+d_{\mathrm{c}} v+d_{\mathrm{j}} v
$$

where the term $d_{\mathrm{a}} v$ is absolutely continuous with respect to $\mathcal{L}^{n}$, the term $d_{\mathrm{c}} v$ (called "Cantor" part of $d v$ ) is singular and does not charge any $\mathcal{H}^{n-1}$-finite set, and the term $d_{\mathrm{j}} v$ (called "jump" part of $d v$ ) is concentrated on a rectifiable set of codimension one. Furthermore the density of $d_{\mathrm{a}} v$ with respect to $\mathcal{L}^{n}$ agrees with the approximate differential of $v$, and $d_{\mathrm{j}} v$ can be written as $d_{\mathrm{j}} v=[v] \eta \mathcal{H}^{n-1}\llcorner S v$, where $S v$ is the singular set (set of point of approximate discontinuity) of $v$, and is $(n-1)$-rectifiable, $\eta$ is the 1-covector associated to an orientation $\tau$ of $S v$ via the $\star$ operator (cf. Sect. 2.7), and the jump [v] is the difference between the traces $v^{+}$ and $v^{-}$of $\theta^{\prime}$ on the two sides of $S$. When the Cantor part $d_{\mathrm{c}} v$ vanishes, $v$ is called an $S B V$ function.

4.3. Construction of for general $M$. Let $M$ be an integral $(n-2)$-current with finite mass in $\mathbb{R}^{n}$, and take an integral $(n-1)$-current $N$ with finite mass such that $M=\partial N$ (cf. Remark 2.6). By formula (3.9), in order to solve the equation $\star J u=\pi M$ it suffices to construct a function $\theta \in W_{\text {loc }}^{1,1}\left(\mathbb{R}^{n}, \mathbb{R} / \mathbb{Z}\right)$ such that $\star d(d \theta)=M$ and take $u:=\exp (2 \pi i \theta)$. As suggested before, such a $\theta$ can be obtained as a suitable convolution product - which is just formula (4.4) re-written.

Let $\omega$ be the pull-back on $\mathbb{R}^{n} \backslash\{0\}$ of the standard volume form on $S^{n-1}$ according to the projection $x \mapsto x /|x|$, that is

$$
\omega(x)=\sum_{i=1}^{n}(-1)^{i-1} \frac{x_{i}}{|x|^{n}} \widehat{d x}_{i} .
$$

Thus $\omega \in L_{\mathrm{loc}}^{p}\left(\mathbb{R}^{n}\right)$ for every $p<1^{*}:=n /(n-1)$, and

$$
d \omega=n \alpha_{n} \delta_{0} d x
$$

where, as usual, $d x=d x_{1} \wedge \cdots \wedge d x_{n} \cdot{ }^{13}$ We set

$$
\theta^{\prime}:=\frac{-1}{n \alpha_{n}} \omega * N,
$$

and, denoting by $\pi$ the canonical projection of $\mathbb{R}$ onto $\mathbb{R} / \mathbb{Z}$,

$$
\theta:=\pi\left(\theta^{\prime}\right) \text {. }
$$

The usual estimates for the convolution product show that $\theta^{\prime}$ is a well-defined real-valued function ( 0 -form) in $L_{\text {loc }}^{p}\left(\mathbb{R}^{n}\right)$ for every $p<1^{*}$, and using (4.7), (2.6), and (4.6), we obtain

$$
d \theta^{\prime}=\frac{(-1)^{n}}{n \alpha_{n}}[d \omega * N+\omega * \partial N]=(-1)^{n}\left[d x \mathbf{L} N+\frac{1}{n \alpha_{n}} \omega * M\right] .
$$

\footnotetext{
13 Indeed $d \omega$ is an $n$-form, smooth outside the origin, whose integral on any open set $A$ with smooth boundary is, by Stokes theorem, the integral of $\omega$ on $\partial A$, that is, $n \alpha_{n}$ (the volume of $S^{n-1}$ ) if the origin belongs to $A$ and 0 otherwise.
} 
Thus $\theta^{\prime}$ is a function of class $B V_{\text {loc }}$ whose differential is the sum of a term absolutely continuous with respect to Lebesgue measure, $\omega * M$, and a jump term of the form $d x \mathrm{~L} N$. Since $N$ has integer multiplicity, the jump of $\theta^{\prime}$ is always integer, and therefore $\theta$ has no jump; thus $\theta$ is of class $W_{\text {loc }}^{1,1} 14$ and $d \theta$ is given by

$$
d \theta=\frac{(-1)^{n}}{n \alpha_{n}} \omega * M
$$

Since $\omega$ is smooth outside the origin, $d \theta$, and $\theta$ as well, are smooth outside the support of $M$. Moreover, using (2.6), (4.6), and taking into account that $\partial M=$ $\partial^{2} N=0$,

$$
d(d \theta)=\frac{1}{n \alpha_{n}}[d \omega * M+\omega * \partial M]=d x \mathrm{~L} M,
$$

and recalling (2.3),

$$
\star d(d \theta)=M .
$$

The following statement summarizes what we have obtained so far.

Theorem 4.4. Let $M$ be a codimension-two integral current with finite mass and no boundary in $\mathbb{R}^{n}$, and set $u:=\exp (2 \pi i \theta)$, where $\theta$ is defined in (4.8). Then $u$ belongs to $W_{\text {loc }}^{1, p}\left(\mathbb{R}^{n}, S^{1}\right)$ for every $p<1^{*}$, it is smooth outside the support of $M$, and satisfies $\star J u=\pi M$.

\section{Maps with prescribed singularity: construction for arbitrary $k$}

In this section we address the converse of Theorem 3.8 in arbitrary codimension, namely the construction of a map $u: \Omega \rightarrow S^{k-1}$ with prescribed Jacobian $\star J u=$ $\alpha_{k} M$, where $M$ is the boundary of a rectifiable current $N$ of codimension $k-1$ in $\Omega$. In Proposition 5.2 we give a simple construction for special $M$, akin to the dipole construction of [14] (see also [21, §4.2.3]), that will provide the building blocks for the construction in the general case. In Theorem 5.6 we prove that any boundary $M$ of a rectifiable current is the Jacobian of a map of class $W^{1, k-1}$. After this general result we examine what further regularity can be obtained on $u$ when $M$ is a polyhedral current (see Theorem 5.10).

14 More precisely, the differential of the complex-valued $S B V$ function $u:=\exp \left(2 \pi i \theta^{\prime}\right)$ con be computed by the chain-rule for $B V$ functions (see for instance [3, Sect. 3.10]):

$$
d u=2 \pi i \cdot \exp \left(2 \pi i \theta^{\prime}\right) \cdot d_{\mathrm{a}} \theta^{\prime}+\left(\exp \left(2 \pi i \theta^{\prime+}\right)-\exp \left(2 \pi i \theta^{\prime-}\right)\right) \cdot \eta \cdot \mathcal{H}^{n-1}\left\llcorner S \theta^{\prime} .\right.
$$

By (4.9) the singular set $S \theta^{\prime}$ is $N$, and the jump $\theta^{\prime+}-\theta^{\prime-}$ is the multiplicity of $N$. Since the latter is integer, $\exp \left(2 \pi i \theta^{\prime+}\right)-\exp \left(2 \pi i \theta^{\prime-}\right)=0$. Thus $u$ is a function of class $W^{1,1}$, and so is $\theta$. 
5.1. Additional notation. For the rest of this section we fix a point $y_{N} \in S^{k-1}$ (called "north pole") and a family of smooth maps $\phi_{\sigma}: S^{k-1} \rightarrow S^{k-1}$ for every $\sigma \in \mathbb{Z}$ with the following properties: $\phi_{0}$ takes constant value $y_{N}, \phi_{1}$ is the identity, $\phi_{-1}$ is a reflection with respect to an hyperplane which contains $y_{N}$, each $\phi_{\sigma}$ has degree $\sigma$, maps $y_{N}$ into itself, and has Lipschitz constant lower than $2|\sigma|{ }^{15}$

Proposition 5.2. Let $P$ be an oriented $(n-k+1)$-dimensional affine subspace of $\mathbb{R}^{n}$, let $N$ be the current in $\mathbb{R}^{n}$ associated with a bounded Lipschitz domain of $P$, endowed with constant multiplicity 1 , and assume that $M:=\partial N$ is connected.

Then there exists $u \in W_{\mathrm{loc}}^{1, k-1}\left(\mathbb{R}^{n}, S^{k-1}\right)$ such that $\star J u=\alpha_{k}$ M. Moreover $u$ is locally Lipschitz in $\mathbb{R}^{n} \backslash M$ and constant outside a bounded neighbourhood of $N$, Du belongs to $L^{p}\left(\mathbb{R}^{n}\right)$ for every $p<k$ and satisfies $|D u(x)|=O(1 / \operatorname{dist}(x, M))$.

Proof. We fix two positive parameters $\delta$ and $\gamma$, and set

$$
U=U(N, \delta, \gamma):=\left\{x: \operatorname{dist}(x, N) \leq \min \left\{\delta, \frac{\gamma}{\sqrt{1+\gamma^{2}}} \operatorname{dist}(x, \partial N)\right\}\right\} .
$$

Now we identify $P$ with $\mathbb{R}^{n-k+1}, \mathbb{R}^{n}$ with the product $\mathbb{R}^{n-k+1} \times \mathbb{R}^{k-1}$, and denote a point in $\mathbb{R}^{n}$ as $x=\left(x^{\prime}, x^{\prime \prime}\right)$. Thus $U$ can be written as (cf. Fig. 2 below)

$$
U=\left\{x: x^{\prime} \in N,\left|x^{\prime \prime}\right| \leq g\left(x^{\prime}\right)\right\},
$$

where

$$
g\left(x^{\prime}\right):=\min \left\{\delta, \gamma \operatorname{dist}\left(x^{\prime}, \partial N\right)\right\} .
$$

Let $B^{k-1}$ be the closed unit ball in $\mathbb{R}^{k-1}$ with center 0 , and let $\pi$ be the usual identification of $B^{k-1}$ into the sphere $S^{k-1}$ which takes the boundary of $B^{k-1}$ into the north pole $y_{N}$ and preserves the orientation ${ }^{16}$, and set

$$
u(x):= \begin{cases}\pi\left(\frac{x^{\prime \prime}}{g\left(x^{\prime}\right)}\right) & \text { for } x \in U \backslash \partial N, \\ y_{N} & \text { elsewhere in } \mathbb{R}^{n} .\end{cases}
$$

Thus $u$ is constant on the complement of $U$.

Since $g$ is a real Lipschitz function which vanishes only on $\partial N$ and $\pi$ is a Lipschitz map from the closed unit ball $B^{k-1}$ to $S^{k-1}$, then the restriction of $u$ to $U \backslash \partial N$ is locally Lipschitz. Moreover $\pi$ takes unit vectors in $y_{N}$, and since $\left|x^{\prime \prime}\right|=g\left(x^{\prime}\right)$ for all $x \in \partial U$, then $u$ maps $\partial U$ into $y_{N}$, and consequently $u \in$ $\operatorname{Lip}_{\text {loc }}\left(\mathbb{R}^{n} \backslash \partial N\right)$.

15 For example, write $\mathbb{R}^{k} \simeq \mathbb{C} \times \mathbb{R}^{k-2}$, where $\mathbb{C}$ is the complex field, take $y_{N}$ of the form $y_{N}=(0, y)$ and, for $\sigma \neq 0$,

$$
\phi_{\sigma}(z, y):= \begin{cases}\left(z^{\sigma}|z|^{1-\sigma}, y\right) & \text { for } z \neq 0 \\ (0, y) & \text { for } z=0 .\end{cases}
$$

16 For example, take $\pi(x):=(\sin (\pi|x|) \cdot x /|x|, \cos (\pi|x|))$ for every $x \in B^{k-1}$, and identify $\mathbb{R}^{k-1} \times \mathbb{R}$ with $\mathbb{R}^{k}$ so that $y_{N}=(0,-1)$. 


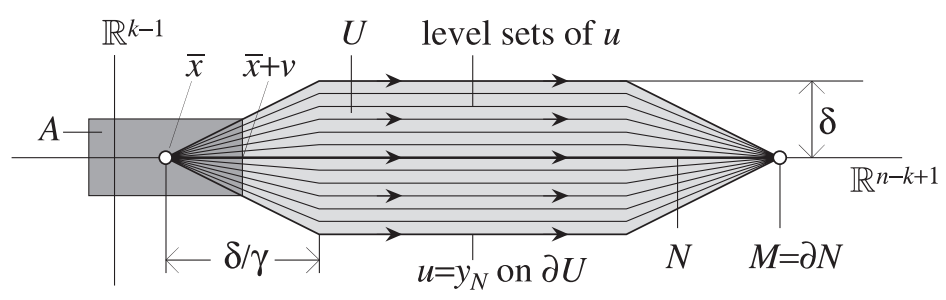

Fig. 2.

To give a precise estimate of the pointwise derivative $|D u|$, we notice that $D g\left(x^{\prime}\right)=0$ when $\gamma \operatorname{dist}\left(x^{\prime}, \partial N\right)>\delta$, and then $\left|D\left(x^{\prime \prime} / g\left(x^{\prime}\right)\right)\right| \leq 1 / \delta$. On the other hand, if $\gamma \operatorname{dist}\left(x^{\prime}, \partial N\right)<\delta$, then $g\left(x^{\prime}\right)=\gamma \operatorname{dist}\left(x^{\prime}, \partial N\right)$ and $\left|D g\left(x^{\prime}\right)\right|=\gamma$, which imply

$$
\left|D\left(x^{\prime \prime} / g\left(x^{\prime}\right)\right)\right| \leq\left(\frac{1}{\gamma}+1\right) \frac{1}{\operatorname{dist}\left(x^{\prime}, \partial N\right)} \leq \frac{(1+\gamma)^{2}}{\gamma \operatorname{dist}(x, \partial N)}
$$

(here we have used that $\operatorname{dist}(x, \partial N) \leq \operatorname{dist}\left(x^{\prime}, \partial N\right)+\left|x^{\prime \prime}\right| \leq(1+\gamma) \operatorname{dist}\left(x^{\prime}, \partial N\right)$, cf. (5.2)). Together with the previous estimate and (5.4), this yields

$$
|D u(x)| \leq\|D \pi\|_{\infty} \max \left\{\frac{1}{\delta}, \frac{(1+\gamma)^{2}}{\gamma \operatorname{dist}(x, \partial N)}\right\},
$$

and the pointwise derivative $D u$ is $p$-summable for every $p<k$ by Lemma 7.1. Since $u$ is locally Lipschitz in $\mathbb{R}^{n} \backslash \partial N$, then the pointwise derivative $D u$ agrees with the distributional derivative on $\mathbb{R}^{n} \backslash \partial N$, and since it is 1 -summable and $N$ has codimension strictly larger than one, $D u$ agrees with the distributional derivative on $\mathbb{R}^{n}$, too. In other words, $u$ is a Sobolev map on $\mathbb{R}^{n}$.

It remains to verify that $\star J u=\alpha_{k} M$, up to a sign that can be corrected by changing the identification of $P$ and $\mathbb{R}^{n-k+1}$. Since $u$ is continuous in the complement of the Lipschitz connected $(n-k)$-surface $M=\partial N$, as remarked in Sect. 3.7 the Jacobian $\star J u$ is supported on $M$, and is of the form $\alpha_{k} \sigma M$ where $\sigma$ is an integer. In view of formula (3.6), to prove that $\sigma= \pm 1$ if suffices to exhibit a $k$-surface $A$ transversal to $M$ such that $A \cap M$ consists just of one point and $\operatorname{deg}\left(u, \partial A, S^{k-1}\right)= \pm 1$.

Take then $\bar{x} \in M$, and $v \in \mathbb{R}^{n-k+1}$, and let $A$ be the cylinder in $\mathbb{R}^{n-k+1} \times \mathbb{R}^{k-1}$ given by the segment $[\bar{x}-v, \bar{x}+v]$ times the closed ball $r B^{k-1}$, where $r:=\gamma|v|$ (see Fig. 2 above). If we have chosen $\bar{x}$ and $v$ so that $(\bar{x}, \bar{x}+v]$ is contained in $N$, $[\bar{x}-v, \bar{x})$ is contained in the complement of $N$, and $x$ is the projection of $\bar{x}+v$ onto $N$, then one easily checks that $u$ is equal to $y_{N}$ on the whole boundary of $A$ except the face $\{\bar{x}+v\} \times r B^{k-1}$, where it agrees, up to rescaling, with the injective map $\pi$. Thus the $\operatorname{deg}\left(u, \partial A, S^{k-1}\right)= \pm 1$, and the proof is complete. 
Corollary 5.3. When the surface $N$ in the statement of Proposition 5.2 is an $(n-k+1)$-dimensional disk $E$ with center $x$ and radius $r$, we may also require that $u$ is constant on the complement of the ball $B(x, r)$ and satisfies

$$
\int_{\mathbb{R}^{n}}|D u|^{k-1} \leq C\|E\|
$$

where $C$ is a constant which depends only on $n$ and $k$.

Proof. Let $u_{0}$ be the map given by Proposition 5.2 when $N$ is a fixed disk $E_{0}$ with center 0 and radius 1 . If the parameters $\delta$ and $\lambda$ in the definition of $U$ (cf. (5.1) and (5.2)) have been chosen sufficiently small, then $U$ is included in $\bar{B}(1)$, and therefore $u_{0}$ is constant on the complement of $B(1)$. Now it suffices to take $u:=u_{0} \circ \psi$, where $\psi$ is an affine similitude - a composition of an isometry and a homothety with scaling factor $r$ which maps $E$ onto $E_{0}$ preserving the orientation.

\section{Construction for general rectifiable currents}

We begin with a Lemma for pasting together $S^{k-1}$-valued maps. In this subsection $\Omega$ is an open subset of $\mathbb{R}^{n}$.

Lemma 5.4. Let $u_{0}, u_{1}$ be maps in $W_{1, k-1}^{1, k-1}\left(\Omega, S^{k-1}\right)$, let $F_{0}, F_{1}$ be disjoint closed sets in $\Omega$, and set $\Omega^{\prime}:=\Omega \backslash\left(F_{0} \cup F_{1}\right)$. If $\operatorname{dist}\left(F_{0}, F_{1}\right)>0$ and

$$
\left|u_{0}-u_{1}\right| \leq 1 \quad \text { a.e. on } \Omega^{\prime},
$$

then there exists $u \in W_{\mathrm{loc}}^{1, k-1}\left(\Omega, S^{k-1}\right)$ such that $u=u_{0}$ in $F_{0}, u=u_{1}$ in $F_{1}$. If $D u_{0}$ and $D u_{1}$ are essentially bounded in the "transition zone" $\Omega^{\prime}$, then $D u$ is essentially bounded on $\Omega^{\prime}$ and

$$
\|D u\|_{L^{\infty}\left(\Omega^{\prime}\right)} \leq \frac{2}{\sqrt{3}}\left[\frac{\left\|u_{0}-u_{1}\right\|_{L^{\infty}\left(\Omega^{\prime}\right)}}{\operatorname{dist}\left(F_{0}, F_{1}\right)}+\left\|D u_{0}\right\|_{L^{\infty}\left(\Omega^{\prime}\right)}+\left\|D u_{1}\right\|_{L^{\infty}\left(\Omega^{\prime}\right)}\right] .
$$

If the Jacobians of $u_{0}$ and $u_{1}$ are supported on $F_{0}$ and $F_{1}$, respectively, then $J u=J u_{0}+J u_{1}$.

Proof. Let $\pi$ be the projection of $\mathbb{R}^{k} \backslash\{0\}$ onto $S^{k-1}$, that is, $\pi(x):=x /|x|$, and take a Lipschitz function $\lambda$ which agrees with 0 on $F_{0}$ and with 1 on $F_{1}$, and has Lipschitz constant smaller than dist ${ }^{-1}\left(F_{0}, F_{1}\right)$, e.g.,

$$
\lambda(x):=\min \left\{1, \frac{\operatorname{dist}\left(x, F_{0}\right)}{\operatorname{dist}\left(F_{0}, F_{1}\right)}\right\} .
$$

Then set

$$
u:=\pi\left(\lambda u_{1}+(1-\lambda) u_{0}\right)
$$

Given $x \in \Omega^{\prime},\left|u_{0}(x)\right|=\left|u_{1}(x)\right|=1$, while $\left|u_{0}(x)-u_{1}(x)\right| \leq 1$ by assumption. Thus the angle spanned by the vectors $u_{0}(x)$ and $u_{1}(x)$ is smaller than $60^{\circ}$, and then every convex combination of $u_{0}(x)$ and $u_{1}(x)$ has norm larger than $\sqrt{3} / 2$. 
This shows that the argument of $\pi$ in formula (5.9) is always contained in $\mathbb{R}^{k} \backslash B^{k}(\sqrt{3} / 2)$, where $\pi$ has Lipschitz constant $2 / \sqrt{3}$. Thus $u$ belongs to $W_{\text {loc }}^{1, k-1}$ too, and a straightforward computation gives (5.8). The last part of the statement is trivial.

Remark 5.5. In the proof of Theorem 5.6 we will apply Lemma 5.4 with $F_{1}=$ $\bar{B}(x, r)$ and $F_{0}=\mathbb{R}^{n} \backslash B(x, 2 r), u_{1}$ constant on the complement of $F_{1}, u_{0}$ continuous in $\Omega^{\prime}=B(x, 2 r) \backslash \bar{B}(x, r)$ and equal to $u_{1}$ at some point of $\Omega^{\prime}$. Under these assumptions we have

$$
\left\|u_{0}-u_{1}\right\|_{L^{\infty}\left(\Omega^{\prime}\right)} \leq \operatorname{osc}\left(u_{0}, \Omega^{\prime}\right) \leq 4 r\left\|D u_{0}\right\|_{L^{\infty}(B(x, 2 r))} .
$$

Hence condition (5.7) is implied by

$$
r \leq\left(4\left\|D u_{0}\right\|_{L^{\infty}(B(x, 2 r))}\right)^{-1},
$$

while inequality (5.8) yields $\|D u\|_{L^{\infty}\left(\Omega^{\prime}\right)} \leq \frac{10}{\sqrt{3}}\left\|D u_{0}\right\|_{L^{\infty}(B(x, 2 r))}$, and then

$$
\int_{\Omega^{\prime}}|D u|^{k-1} \leq\left(\frac{10}{\sqrt{3}}\left\|D u_{0}\right\|_{L^{\infty}(B(x, 2 r))}\right)^{k-1} 2^{n} \alpha_{n} r^{n} \leq C r^{n-k+1},
$$

where the last inequality follows from (5.10), and $C$ is a constant which depends on $n$ and $k$ only.

The following statement contains the exact converse of Theorem 3.8; for $k=2$ there exists a simpler proof which is briefly sketched in Sect. 6.7.

Theorem 5.6. Let $\Omega$ be an open set in $\mathbb{R}^{n}$, and $M$ the boundary of a rectifiable current $N$ in $\Omega$ with finite mass and codimension $k-1$. Then there exists $u$ in $W_{\text {loc }}^{1, k-1}\left(\Omega, S^{k-1}\right)$ such that

$$
\star J u=\alpha_{k} M
$$

Moreover $\|D u\|_{k-1} \leq C\|N\|$, where $C$ depends only on $n$ and $k$. In particular $u$ belongs to $W^{1, k-1}\left(\Omega, S^{k-1}\right)$ when $\Omega$ has finite measure.

Proof. We define, by induction on $j$, a sequence of maps $u_{j} \in W^{1, k-1}\left(\Omega, S^{k-1}\right)$ which are locally Lipschitz on the complement of a closed sets $S_{j} \subset \Omega$ of codimension $k$, and rectifiable currents $R_{j}$ in $\Omega$ of codimension $k-1$ so that $R_{0}=N$, $D u_{0}=0, S_{0}=\varnothing$ and

$$
\begin{aligned}
& \star J u_{j}=\alpha_{k}\left(M-\partial R_{j}\right), \\
& \left\|D u_{j}-D u_{j-1}\right\|_{k-1} \leq C\left\|R_{j-1}\right\|^{1 /(k-1)}, \\
& \left\|R_{j}\right\| \leq\left(1-2^{k-n-2}\right)\left\|R_{j-1}\right\|,
\end{aligned}
$$

where $C$ depends only on $n$ and $k$.

We first show that (5.13-5.15) allow us to conclude the proof. Inequality (5.15) implies $\left\|R_{j}\right\| \leq\left(1-2^{k-n-2}\right)^{j}\left\|R_{0}\right\|$, which is a summable sequence in $j$. Hence 
(5.14) shows that the derivatives $D u_{j}$ form a Cauchy sequence in $L^{k-1}$ and therefore the maps $u_{j}$ converge (up to subsequence) to some map $u$ in the strong topology of $W_{\text {loc }}^{1, k-1}\left(\Omega, S^{k-1}\right)$. Hence $J u_{j}$ converge to $J u$ in the sense of distributions (cf. Remark 3.4). On the other hand, identity (5.13) shows that $\star J u_{j}$ converge to $\alpha_{k} M$ in the sense of currents (because $R_{j}$ converge to 0 in norm), and (5.12) is proved. The estimate on $\|D u\|_{k-1}$ follows from (5.14), (5.15) and the fact that $R_{0}=N$ and $D u_{0}=0$.

Description of the inductive procedure. Given $u_{j-1}, S_{j-1}$, and $R_{j-1}$, we construct $u_{j}, S_{j}$, and $R_{j}$. Since $S_{j-1}$ is closed, for every $x \in \Omega \backslash S_{j-1}$ we can find $r_{j}=$ $r_{j}(x)>0$ such that $B\left(x, r_{j}\right)$ is relatively compact in $\Omega \backslash S_{j-1}$. Since $u_{j-1}$ is locally Lipschitz on the complement of $S_{j-1},\left|D u_{j-1}\right|$ is essentially bounded in $B\left(x, r_{j}\right)$. We choose $\rho_{j}: \Omega \rightarrow[0,+\infty)$ so that $\rho_{j}(x)=0$ for $x \in S_{j-1}$ and

$$
0<\rho_{j} \leq \min \left\{\frac{r_{j}}{2}, \frac{1}{4\left\|D u_{j-1}\right\|_{L^{\infty}\left(B\left(x, r_{j}\right)\right)}}\right\} \text { for } x \in \Omega \backslash S_{j-1} .
$$

Since $S_{j-1}$ is $\mathcal{H}^{n-k+1}$-negligible, we can apply Corollary 7.13 with $h:=n-k+1$, $N:=R_{j-1}$ and $\rho:=\rho_{j}$, and get finitely many disks $E_{j i}$, with centers $x_{j i} \in \Omega$ and radii $r_{j i}<\rho_{j}\left(x_{j i}\right)$, and rectifiable currents $R_{j}$ and $P_{j}$ which satisfy statements (i)-(iv) of Corollary 7.13. In particular (iii) implies (5.15).

Then for every $i$ we take a map $u_{j i}$ according to Corollary 5.3 for $N:=E_{j i}$. Thus $u_{j i}$ is constant on the complement of $B\left(x_{j i}, r_{j i}\right)$, and, possibly composing $u_{j i}$ by a suitable isometry of $S^{k-1}$, we can assume that this constant agrees with the value of $u_{j-1}$ at some point of the annulus $B\left(x_{j i}, 2 r_{j i}\right) \backslash \bar{B}\left(x_{j i}, r_{j i}\right)$. Now we use Lemma 5.4 to construct a map $u_{j}$ such that

$$
u_{j}=u_{j i} \text { on } B\left(x_{j i}, r_{j i}\right) \text { for every } i \text {, and } u_{j}=u_{j-1} \text { on } \Omega \backslash \bigcup_{i} B\left(x_{j i}, 2 r_{j i}\right) \text {. }
$$

Indeed, the balls $\bar{B}\left(x_{j i}, 2 r_{j i}\right)$ are disjoint by statement (iv) of Corollary 7.13, the inequalities $r_{j i}<\rho_{j}\left(x_{j i}\right)$ and (5.16) imply that $u_{j-1}$ satisfies condition (5.10) in Remark 5.5, while $u_{j i}$ is constant on the complement of $B\left(x_{j i}, r_{j i}\right)$ by construction, and then Lemma 5.4 can be applied.

Verification of the properties of $u_{j}$. By construction, the map $u_{j}$ belongs to $W_{\text {loc }}^{1, k-1}(\Omega)$ and is locally Lipschitz on the complement of

$$
S_{j}:=S_{j-1} \cup\left(\bigcup_{i} \partial E_{j i}\right) .
$$

Moreover (cf. Lemma 5.4)

$$
J u_{j}=J u_{j-1}+\sum_{i} J u_{j i} .
$$

On the other hand, we have chosen $u_{j i}$ so that $\star J u_{j i}=\alpha_{k} \partial E_{j i}$ (cf. Proposition 5.2), while $\sum \partial E_{j i}=\partial R_{j-1}-\partial R_{j}$ by condition (i) of Corollary 7.13 and $\star J u_{j-1}=$ 
$\alpha_{k}\left(M-\partial R_{j-1}\right)$ by the inductive assumption (5.13) for $j-1$. Thus (5.17) yields (5.13) for $j$.

It remains to prove (5.14). If we denote by $\Omega^{\prime}$ the union of all transition zones $B\left(x_{j i}, 2 r_{j i}\right) \backslash \bar{B}\left(x_{j i}, r_{j i}\right)$, then $u_{j}$ satisfies

$$
\int_{\Omega^{\prime}}\left|D u_{j}\right|^{k-1} \leq C \sum_{i} r_{j i}^{n-k+1} \leq \frac{C}{\alpha_{n-k+1}} \sum_{i}\left\|E_{j i}\right\| \leq \frac{C 2^{k-n}}{\alpha_{n-k+1}}\left\|R_{j-1}\right\|,
$$

where the first inequality follows from (5.11), and the third one from statement (ii) of Corollary 7.13 (with $R_{j-1}$ playing the rôle of $N$ ). On the other hand, estimate (5.6) and the fact that $u_{j}=u_{j i}$ on $B\left(x_{j i}, r_{j i}\right)$ yield

$$
\sum_{i} \int_{B\left(x_{j i}, r_{j i}\right)}\left|D u_{j}\right|^{k-1} \leq C \sum_{i}\left\|E_{j i}\right\| \leq C 2^{k-n}\left\|R_{j-1}\right\|,
$$

while (5.16) and the fact that $r_{j i} \leq \rho\left(x_{j i}\right)$ give

$$
\begin{aligned}
\sum_{i} \int_{B\left(x_{j i}, 2 r_{j i}\right)}\left|D u_{j-1}\right|^{k-1} \leq \alpha_{n} 2^{n-2 k+2} \sum_{i} r_{j i}^{n-k+1} \\
=\frac{\alpha_{n} 2^{n-2 k+2}}{\alpha_{n-k+1}} \sum_{i}\left\|E_{j i}\right\| \leq \frac{\alpha_{n} 2^{2-k}}{\alpha_{n-k+1}}\left\|R_{j-1}\right\| .
\end{aligned}
$$

Estimates (5.18-5.20) imply (5.14).

\section{Construction for polyhedral currents}

When $M$ is a polyhedral current, we can use Proposition 5.2 to construct a map $u$ which is more regular than that provided by Theorem 5.6. To this end we need the following:

Lemma 5.7. Let $p \geq k-1$, and let be given finitely manymaps $u_{i} \in W_{1 \text { loc }}^{1, p}\left(\mathbb{R}^{n}, S^{k-1}\right)$ which are locally Lipschitz in the complement of closed sets $S_{i}$ and equal to the north pole $y_{N}$ in the complement of bounded open sets $\Omega_{i}$.

If the sets $\Omega_{i}$ are disjoint and $u$ is the map which agrees with $u_{i}$ on each $\Omega_{i}$, and takes the value $y_{N}$ elsewhere in $\mathbb{R}^{n}$, then $u$ belongs to $W_{\text {loc }}^{1, p}\left(\mathbb{R}^{n}, S^{k-1}\right)$, is locally Lipschitz in the complement of $\cup S_{i}$, and $J u=\sum_{i} J u_{i}$.

Proof. It is clear that $u$ is locally Lipschitz on $\mathbb{R}^{n} \backslash \cup S_{i}$. The rest of the statement is trivial if the sets $\Omega_{i}$ have disjoint closures, and the general case follows by approximation.

Proposition 5.8. Let $M$ be the boundary of a polyhedral current $N$ of codimension $k-1$ in $\mathbb{R}^{n}$, and denote by $\Sigma$ the union of the faces of $N$ of codimension $k$. Then there exists a map $u \in W_{\mathrm{loc}}^{1, k-1}\left(\mathbb{R}^{n}, S^{k-1}\right)$ such that $\star J u=\alpha_{k}$ M. Moreover $u$ is locally Lipschitz in $\mathbb{R}^{n} \backslash \Sigma$ and constant outside a bounded neighbourhood of $N$, and $D u$ belongs to $L^{p}\left(\mathbb{R}^{n}\right)$ for every $p<k$ and satisfies $|D u(x)|=O(1 / \operatorname{dist}(x, \Sigma))$. 
Proof. Write $N$ as the finite sum $\sum \sigma_{i} N_{i}$, where each $N_{i}$ is a simplex (endowed with multiplicity 1), and $\sigma_{i}$ is an integer. We assume as usual that $N_{i} \cap N_{j}$ is either empty or a common face of $N_{i}$ and $N_{j}$, and then we can find $\delta, \gamma>0$ such that the sets $U_{i}:=U\left(N_{i}, \delta, \gamma\right)$ defined in (5.1) have pairwise disjoint interiors (see Fig. 3).

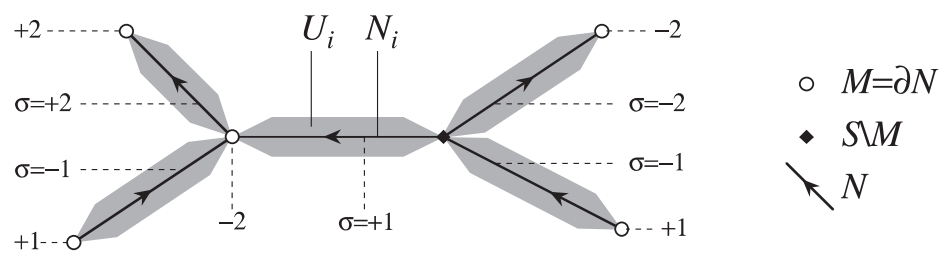

Fig. 3.

For every $i$, take $u_{i}$ according to Proposition 5.2 for $N:=N_{i}$, set $\tilde{u}_{i}:=\phi_{\sigma_{i}} \circ u_{i}$ where $\phi_{\sigma_{i}}$ is taken as in Sect. 5.1, and let $u$ be the map which agrees with $\tilde{u}_{i}$ on the interior of each $U_{i}$, and with $y_{N}$ elsewhere in $\mathbb{R}^{n}$. By Corollary, 3.9, $\star J \tilde{u}_{i}=$ $\alpha_{k} \sigma_{i} \partial N_{i}$ for every $i$, and $\star J u=\alpha_{k} \sum \sigma_{i} N_{i}=\alpha_{k} M$ by Lemma 5.7.

The previous result gives a map $u$ with Jacobian $\star J u=\alpha_{k} M$, which is singular on the union $\Sigma$ of the $(n-k)$-dimensional faces of $N$. Notice that $\Sigma$ contains the support of $M=\partial N$, but in general can be larger (see Fig. 3). In other words, part of the singularity of $u$ supports no Jacobian. In the next paragraph we describe a procedure to remove some (but not all) of this "unnecessary" singularity, and optimize the singularity around $M$.

5.9. Optimization of singularities. Let $M, N, \Sigma$ and $u$ be given as in the proof of Proposition 5.8. Let $F$ be an $(n-k)$-dimensional face of $N$, endowed with some orientation, and $\sigma$ the multiplicity of $M=\partial N$ on $F$ (thus $\sigma=0$ if $F$ is not contained in the support of $\partial N$ ).

In the following we identify $\mathbb{R}^{n}$ with the product $\mathbb{R}^{n-k} \times \mathbb{R}^{k}$, denoting a point in $\mathbb{R}^{n}$ as $x=\left(x^{\prime}, x^{\prime \prime}\right)$, and assume that $F$ lies on $\mathbb{R}^{n-k}$. Then we set (cf. (5.2) and (5.3))

$$
U^{\prime}=U^{\prime}\left(F, \delta^{\prime}, \gamma^{\prime}\right):=\left\{x: x^{\prime} \in F,\left|x^{\prime \prime}\right| \leq g^{\prime}\left(x^{\prime}\right)\right\}
$$

where $g^{\prime}\left(x^{\prime}\right):=\min \left\{\delta^{\prime}, \gamma^{\prime} \operatorname{dist}\left(x^{\prime}, \partial F\right)\right\}$, and the parameters $\delta^{\prime}, \gamma^{\prime}>0$ are chosen small enough to have $\delta^{\prime}<\delta / \gamma$, where $\delta, \gamma$ are the parameters in the proof of Proposition 5.8, and (see Fig. 4 below)

$$
\operatorname{dist}(x, \Sigma)=\operatorname{dist}(x, F) \text { for every } x \in U^{\prime} .
$$

We claim that within $U^{\prime}$, the map $u$ depends only on $x^{\prime \prime} /\left|x^{\prime \prime}\right|$, that is, it can be written as $u(x):=\phi^{\prime}\left(x^{\prime \prime} /\left|x^{\prime \prime}\right|\right)$ for some Lipschitz map $\phi^{\prime}: S^{k-1} \rightarrow S^{k-1}$. Let indeed $N_{i}$ be any of the $(n-k+1)$-faces of $N$ whose boundary contains $F$, 


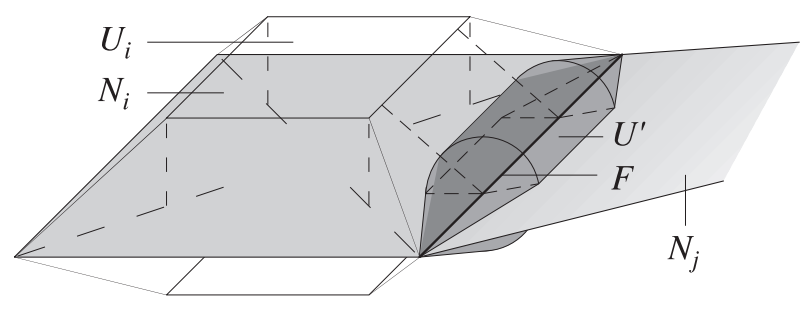

Fig. 4.

and let $U_{i}$ be the corresponding set defined in (5.1). In $U^{\prime} \cap U_{i}, u$ is defined by formula $(5.4)^{17}$. Now, the inner normal to $\partial N_{i}$ agrees on $F$ with a vector which lies in $\{0\} \times \mathbb{R}^{k}$, and we can freely assume that it agrees with the $(n-k+1)$-th element of the canonical basis of $\mathbb{R}^{n}$. Hence, writing $x^{\prime \prime} \in \mathbb{R}^{k}$ as $x^{\prime \prime}=\left(x_{1}^{\prime \prime}, x_{2}^{\prime \prime}\right) \in \mathbb{R} \times \mathbb{R}^{k-1}$, formula (5.4) becomes

$$
u(x)=\pi\left(\frac{x_{2}^{\prime \prime}}{g\left(x^{\prime}, x_{1}^{\prime \prime}\right)}\right) \text { for all } x \in U^{\prime} \cap U_{i} .
$$

Moreover, (5.22) implies $g\left(x^{\prime}, x_{1}^{\prime \prime}\right)=\gamma \operatorname{dist}\left(x, \partial N_{i}\right)=\gamma \operatorname{dist}(x, F)=\gamma x_{1}^{\prime \prime}$ for every $x \in U^{\prime}$, therefore $u(x)=\pi\left(\gamma^{-1} x_{2}^{\prime \prime} / x_{1}^{\prime \prime}\right)$, and the claim is proved.

Since $\star J u=\alpha_{k} M$ and $M$ has multiplicity $\sigma$ on $F$, then the degree of $\phi^{\prime}$ must be $\sigma$ (recall Sect. 3.7). And since the degree classifies the homotopy classes of maps from any $(k-1)$-dimensional compact manifold without boundary into $S^{k-1}$ (Hopf theorem, see for instance [25, Chap. 5, Theorem 1.10]), then $\phi^{\prime}$ must be homotopic to $\phi_{\sigma}$, that is, there exists a Lipschitz map $\Phi: S^{k-1} \times[0,1] \rightarrow S^{k-1}$ such that $\Phi(\cdot, 0)=\phi_{\sigma}(\cdot)$ and $\Phi(\cdot, 1)=\phi^{\prime}(\cdot)$. Obviously, we can also require that $\Phi(\cdot, t)=\phi_{\sigma}(\cdot)$ for all $t \leq 1 / 2$. Finally we set (cf. Fig. 5)

$$
u^{\prime}(x):= \begin{cases}u(x) & \text { when }\left|x^{\prime \prime}\right|>g^{\prime}\left(x^{\prime}\right), \\ \Phi\left(\frac{x^{\prime \prime}}{\left|x^{\prime \prime}\right|}, \frac{\left|x^{\prime \prime}\right|}{g^{\prime}\left(x^{\prime}\right)}\right) & \text { when }\left|x^{\prime \prime}\right| \leq g^{\prime}\left(x^{\prime}\right) .\end{cases}
$$

Thus $u^{\prime}$ agrees with $u$ on the complement of $U^{\prime}$, and with $\phi_{\sigma}\left(x^{\prime \prime} /\left|x^{\prime \prime}\right|\right)$ on $U^{\prime \prime}:=$ $U^{\prime}\left(F, \delta^{\prime} / 2, \gamma^{\prime} / 2\right)$ (see definition (5.21)). In particular, if $\sigma=0$ then $u^{\prime}$ is constant on this set. As in the proof of Proposition 5.2, one easily verifies that $u^{\prime}$ is a Sobolev map from $\mathbb{R}^{n} \rightarrow S^{k-1}$ which is locally Lipschitz on the complement of $\Sigma$ when $\sigma \neq 0$, and on the complement of $(\Sigma \backslash F) \cup \partial F$ when $\sigma=0$. The derivative of $u^{\prime}$ satisfies $D u^{\prime} \in L^{p}\left(\mathbb{R}^{n}\right)$ for every $p<k$ and $\left|D u^{\prime}\right|=O(1 / \operatorname{dist}(x, \Sigma))$ when $\sigma \neq 0,\left|D u^{\prime}\right|=O(1 / \operatorname{dist}(x,(\Sigma \backslash F) \cup \partial F))$ when $\sigma=0$. Finally, $\star J u^{\prime}=\star J u=$ $\alpha_{k} M$.

If we iterate this construction for all $(n-k)$-dimensional faces of $N$ we immediately obtain the following improved version of Proposition 5.8:

17 Note that the variables $x^{\prime}$ and $x^{\prime \prime}$ in formula (5.4) have a different meaning than here. 

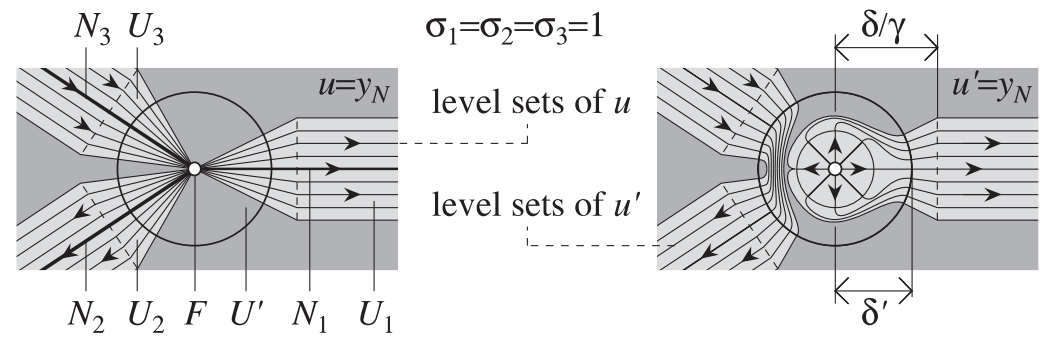

Fig. 5.

Theorem 5.10. Let $M$ be the boundary of a polyhedral current $N$ of codimension $k-1$ in $\mathbb{R}^{n}$, and let $S$ denote the union of the faces of $N$ of codimension $k+1$. Then there exists $u \in W_{\mathrm{loc}}^{1, k-1}\left(\mathbb{R}^{n}, S^{k-1}\right)$ such that $\star J u=\alpha_{k} M, u$ is locally Lipschitz in the complement of $M \cup S$ and constant outside a bounded neighbourhood of $N, D u$ belongs to $L^{p}\left(\mathbb{R}^{n}\right)$ for every $p<k$ and satisfies $|D u(x)|=O(1 / \operatorname{dist}(x, M \cup S))$.

Moreover, given a face $F$ of $M$ of codimension $k$, if we identify the affine plane of codimension $k$ which contains $F$ with $\mathbb{R}^{n-k}$, and write $x \in \mathbb{R}^{n}$ as a $x=\left(x^{\prime}, x^{\prime \prime}\right) \in \mathbb{R}^{n-k} \times \mathbb{R}^{k}$, then

$$
u(x)=\phi_{\sigma}\left(\frac{x^{\prime \prime}}{\left|x^{\prime \prime}\right|}\right) \quad \text { for all } x \in U^{\prime}(F, \delta, \gamma),
$$

where $\sigma$ is the multiplicity of $M$ on $F, \phi_{\sigma}$ is defined in Sect. 5.1, and $U^{\prime}(F, \delta, \gamma)$ is defined as in (5.21) for suitable $\delta$ and $\gamma$.

\section{Additional remarks and open problems}

We collect in this section, with no precise order, some remarks and questions related to the previous sections. We being with an open problem on the structure of Jacobians.

6.1. Pointwise characterization of the Jacobian. There is a clear analogy between Corollary 3.10 and the structure theorem for finite perimeter sets of De Giorgi. Indeed, for $k=1$ the maps from $\Omega$ into $S^{k-1}$ reduce to characteristic functions of sets, while the Jacobian is just the distributional derivative. To this regard, we recall that the rectifiable set which carries the derivative of the characteristic function of a finite perimeter set $E$ can be pointwise characterized as the measuretheoretic boundary of $E$, namely the set of points where $E$ has neither density 1 nor density 0 (see for instance [3, Theorem 3.61] or [17, Theorem 4.5.6(3)] - in fact, finite perimeter sets can be characterized via the measure of their measure-theoretic boundary, cf. [17, Theorem 4.5.11]).

It would be interesting to know if there exists an analogous characterization of the rectifiable set which carries the Jacobian of an $S^{k-1}$-valued map. 
6.2. Algebraic structure of the Jacobian. The space $W^{1,1}\left(\Omega, S^{1}\right)$ is a group under complex multiplication, and it is easy to check that the operator $\star J$ defines, up to a factor $\pi$, a homomorphism of this group into the group of boundaries of rectifiable currents in $\Omega$ with finite mass (use the isomorphism of $W^{1,1}\left(\Omega, S^{1}\right)$ onto $W^{1,1}(\Omega, \mathbb{R} / \mathbb{Z})$, identity (3.9), and the fact that $\theta \mapsto d(d \theta)$ is obviously additive). Theorem 5.6 shows that this homomorphism is also surjective.

Notice moreover that in Sect. 4.3 we (almost) construct an explicit rightinverse of the homomorphism $\star J$ : indeed the map which takes $M$ into $d \theta$ described in (4.10) is linear and satisfies $\star d(d \theta)=M$, and therefore the map which takes $M$ into $u:=\exp (2 \pi i \theta)-$ modulo multiplication by a unit complex number - is a homomorphism, and satisfies $\star J u=\pi M$, that is, it is a right inverse of $\star J$ (but it may be not defined when $M$ has not locally finite mass, cf. Sect. 6.3).

In the next paragraphs we examine the regularity properties of the map $u$ in Theorem 4.4. Thus $M, \theta$ and $u$ are defined as in Sect. 4.3 and Theorem 4.4.

6.3. Relation with the Laplace equation. If $\omega$ is the $(n-1)$-form in (4.5), then the vectorfield $\star \omega$ agrees, up to some constant, with the gradient of the function $|x|^{n-2}$ $(\log |x|$ for $n=2)$, which is the fundamental solution of the Laplace equation on $\mathbb{R}^{n}$. Therefore, if we identify $N$ with a vector measure $\mu$ via the Hodge operator, the function $\theta^{\prime}$ in (4.7) agrees, up to some constant, with the divergence of the solution $v$ of $\Delta v=\mu$.

Now it is well-known that if $v$ solves the Laplace equation with measure or even $L^{1}$ right-hand side, then the second derivative $D^{2} v$ may be not a measure, and the best Sobolev regularity one can expect is, in general, $v \in W^{1, p}$ for every $p<1^{*}$ (see [4] for examples in the context of measure forms).

This remark suggests (but does not prove) that without the assumption that $M=\partial N$ has finite mass, the gradient $D v$, and perhaps also $\theta^{\prime}=\operatorname{div} v$, may be not $B V$ functions. In particular, the function $\theta$ in (4.8) may be not $W^{1,1}$, and then the construction in Sect. 4.2 fails. On the other hand, if $M$ has finite mass, the same argument applied to the convolution product at the right-hand side of (4.10) shows that the Sobolev regularity we have obtained for $\theta$ and $u$ is probably optimal.

6.4. Behaviour close to the singularity. Let $M$ be a plane of codimension two; if we identify $M$ with $\mathbb{R}^{n-2}$, and write $x \in \mathbb{R}^{n}$ as $x=\left(x^{\prime}, x^{\prime \prime}\right) \in \mathbb{R}^{n-2} \times \mathbb{R}^{2}$, then an explicit computation shows that the map $u$ in Theorem 4.4 agrees, up to a rotation in $S^{1}$ which depends on the choice of $N$, with $x^{\prime \prime} /\left|x^{\prime \prime}\right|$.

Using this fact one can show that when $M$ is a smooth surface, $u$ displays a similar behaviour in the proximity of $M$. In particular, it is possible to modify $u$ in a neighbourhood of $M$ so that it remains smooth in the complement of $M$, and for every $x_{0} \in M$ there exist a neighbourhood $U$ of $x_{0}$ and a diffeomorphism $\Psi$ which maps $U$ into the product $M \times \mathbb{R}^{2}$, so that $u(x)=x^{\prime \prime} /\left|x^{\prime \prime}\right|$ for all $x \in U$, where $\left(x^{\prime}, x^{\prime \prime}\right)=\Psi(x)$.

6.5. Geometric consequences. Let $u$ be the map modified as in the previous paragraph, and $y$ a regular value of $u$, then $M$ is the boundary of the smooth 
hypersurface $N:=u^{-1}(y)$. Moreover, if $\left(y_{1}, y_{1}^{\prime}\right)$ and $\left(y_{2}, y_{2}^{\prime}\right)$ are two couples of antipodal regular values of $u$, then $N_{1}:=u^{-1}\left(y_{1}\right) \cup u^{-1}\left(y_{1}^{\prime}\right)$ and $N_{2}:=$ $u^{-1}\left(y_{2}\right) \cup u^{-1}\left(y_{2}^{\prime}\right)$, suitably oriented, are smooth, transversal hypersurfaces without boundary, and $M=N_{1} \cap N_{2}$.

In other words, every smooth surface of codimension two and without boundary in $\mathbb{R}^{n}$ is the boundary of a smooth hypersurface, and even more, it is a complete intersection; notice that these results do not hold in codimension larger than two (see [9], and references therein).

The next paragraphs are devoted to the construction described in Sect. 5.

Remark 6.6. The construction in the proof of Proposition 5.2 can be extended, with few straightforward modifications, to every surfaces $N$ of class $C^{1}$ with trivial normal bundle in $\mathbb{R}^{n}{ }^{18}$ and yields a map $u$ which satisfies $\star J u=\alpha_{k} M$ and is smooth in the complement of $M-\mathrm{cf}$. Theorem 5.10. This triviality condition is automatically satisfied by every oriented surface $N$ of codimension one, and such an $N$ can be found for every $M$ of codimension two (cf. Sect. 6.5).

6.7. A simple proof of Theorem 5.6 for $k=2$. Let $M$ be the boundary in $\Omega$ of a rectifiable current $N$ of codimension one. Then there exists a real function $\theta^{\prime} \in S B V(\Omega)$ whose differential can be decomposed as $\star d \theta^{\prime}=\star \omega+(-1)^{n} N$, where $\omega$ is a suitable 1 -form in $L^{1}(\Omega) .{ }^{19}$ Now we set $\theta:=\pi\left(\theta^{\prime}\right)$, where $\pi$ is the canonical projection of $\mathbb{R}$ onto $\mathbb{R} / \mathbb{Z}$. Since $N$ has integral multiplicity, the jump of $\theta^{\prime}$ is integer, and then $\theta$ has no jump. Hence $\theta$ belongs to $W^{1,1}(\Omega, \mathbb{R} / \mathbb{Z})$ and $\star d \theta=\star \omega=\star\left(d \theta^{\prime}\right)+(-1)^{n-1} N$, and therefore, using (2.2) and recalling that $\partial N=M$ and $d^{2} \theta^{\prime}=0$,

$$
\star d(d \theta)=\star d\left(d \theta^{\prime}\right)+\partial N=M .
$$

By (3.9), the map $u=\exp (2 \pi i \theta)$ satisfies $\star J u=\pi d(d \theta)=\pi M$.

6.8. Is it possible to reduce the singular set of $u$ ? The singular set of the map $u$ in Theorem 5.10 is the union of the datum $M$ and an additional polyhedral set $S$ of codimension $k+1$ (disjoint from $M$ ). It is then natural to ask if $S$ can be removed, or at least replaced by a set of lower dimension. To this end, it is conceivable to adapt the procedure described in Paragraph 5.13: given a face $F$ of dimension $n-k+1$ of $S$, we take a set $U^{\prime}$ as in (5.21), so that $u$ is continuous on $U^{\prime} \backslash F$, and try to replace $u$ inside $U^{\prime} \backslash \partial F$ with a continuous extension of its

\footnotetext{
18 The triviality of the normal bundle implies that some neighbourhood of $N$ is diffeomorphic to the product $N \times \mathbb{R}^{k-1}$.

19 This claim is a particular case of the following statement: given an oriented $(n-1)$ rectifiable set $S$ in $\Omega$ and a real function $g$ in $L^{1}\left(\mathcal{H}^{n-1}\llcorner S)\right.$, there exists an $S B V$ function $v$ such that $S v=S,[v]=g$ and $\left\|d_{a} v\right\| \leq 2\|g\|_{1}$ (cf. [17, §4.1.18]). Indeed, when $S$ is (contained in) the boundary of a domain $A$ of class $C^{1}$ relatively compact in $\Omega$, it suffices to apply a well-known result of E. Gagliardo [18] to find $v \in W^{1,1}(A)$ with $\|d v\|_{1} \leq 2\|g\|_{1}$ and trace equal to $g$ on $\partial A$, and then extend $v$ to 0 on the rest of $\Omega$. The general case can be reduced to this one by covering $S$ with countably many boundaries of class $C^{1}$.
} 
restriction to $\partial U^{\prime} \backslash \partial F$. Now $U^{\prime} \backslash \partial F$ is homeomorphic to the product $F^{\prime} \times \bar{B}^{k+1}$, where $F^{\prime}$ is the interior of $F$ and $\bar{B}^{k+1}$ is the unit closed ball in $\mathbb{R}^{k+1}$, and the problem becomes to find a continuous extension of $u: F^{\prime} \times \partial \bar{B}^{k+1} \rightarrow S^{k-1}$ to $F^{\prime} \times \bar{B}^{k+1}$. Since $F^{\prime}$ is a contractible space, this can be done if and only if, taken an arbitrary point $x^{\prime} \in F^{\prime}$, the restriction of $u$ to $\left\{x^{\prime}\right\} \times \partial B^{k+1}$ (which is homeomorphic to $S^{k}$ ) is homotopic to a constant map. Unfortunately, the $k$-th homotopy group of $S^{k-1}$ is not trivial for $k>2,{ }^{20}$ and therefore such a homotopy may not exist (for a general overview of topological obstruction problems, see for instance [36, Part III]).

Thus we do not know if, and at which cost (in terms of regularity) $S$ can be removed. However, we know that for $k>2$ there cannot always exists a map $u$ which is smooth in the complement of $M$ and behaves close to the singularity $M$ as regularly as the one in Sect. 6.4, because the geometric consequences described in Sect. 6.5 do not hold in codimension larger than two.

\section{Appendix}

We begin this section with a statement on the integrability of certain singular functions. Then we define the notion of Jacobians for Sobolev maps valued in some manifold, and give a version of the coarea formula for such maps; this is the key lemma in the proof Theorem 3.8. We conclude with an approximation result for rectifiable currents.

Lemma 7.1. Let $S \subset \mathbb{R}^{n}$ be (contained in) a finite union of Lipschitz surfaces of codimension $h$. Then, given a bounded set $E \subset \mathbb{R}^{n}$,

$$
\int_{E} \frac{d x}{[\operatorname{dist}(x, S)]^{p}}<+\infty \text { for every } p<h
$$

Proof. Let $M$ be the supremum of $\operatorname{dist}(x, S)$ for $x \in E$, and for every $t>0$ denote by $S_{t}$ the $t$-neighbourhood of $S$, and by $1_{t}$ its characteristic function. Then

$$
\frac{1}{[\operatorname{dist}(x, S)]^{p}}=M^{-p}+p \int_{0}^{M} t^{-(p+1)} 1_{t}(x) d t \quad \text { for every } x \in \mathbb{R}^{n} .
$$

Since $\mathcal{L}^{n}\left(S_{t}\right) \leq C t^{h}$ for some finite $C$ (see [17, §3.2.39]),

$$
\begin{aligned}
\int_{E} \frac{d x}{[\operatorname{dist}(x, S)]^{p}} & =\mathcal{L}^{n}(E) M^{-p}+\int_{0}^{M} \frac{p}{t^{p+1}} \mathcal{L}^{n}\left(S_{t}\right) d t \\
& \leq \mathcal{L}^{n}(E) M^{-p}+C p \int_{0}^{M} t^{h-1-p} d t<+\infty
\end{aligned}
$$

$\overline{20}$ In particular $\pi_{3}\left(S^{2}\right)=\mathbb{Z}$ and $\pi_{k}\left(S^{k-1}\right)=\mathbb{Z}_{2}$ for $k \geq 3$, see for instance [13, Chap. VII, Theorem 8.3]. 
7.2. Pull-back according to Sobolev maps. Let $\Omega$ be an open subset of $\mathbb{R}^{n}$, and $\omega$ a fixed smooth $h$-form in $\mathbb{R}^{k}$, with $h \leq k \leq n$. For every $C^{1}$ map $u: \Omega \rightarrow \mathbb{R}^{k}$, we denote by $J_{\omega} u$ the pull-back of $\omega$ according to $u$ (we adopt this notation to mark the analogy with the Jacobian). Since

$$
\left|J_{\omega} u(x)\right| \leq|\omega(u(x))| \cdot|D u(x)|^{h},
$$

this pointwise definition makes sense (almost everywhere) for every bounded map of class $W^{1, p}$ with $p \geq h$, and $J_{\omega} u$ belongs to $L^{p / h}$. Moreover the operator $J_{\omega}$ is continuous, in the sense that given a sequence of uniformly bounded maps $u_{j}$ that converge to $u$ strongly in $W^{1, p}$, the Jacobians $J_{\omega} u_{j}$ converge to $J_{\omega} u$ strongly in $L^{p / h}$.

The pull-back according to a map $u$ of class $C^{2}$ commutes with the exterior differential, that is

$$
J_{d \omega} u=d\left(J_{\omega} u\right)
$$

and therefore, if $\psi$ is the differential of an $h$-form $\omega$, for all $u$ of class $C^{2}$ there holds

$$
J_{\psi} u=d\left(J_{\omega} u\right)
$$

Since $J_{\omega} u$ is well-defined for all bounded maps of class $W^{1, h}$, formula (7.3) allows us to define $J_{\psi} u$ as a distribution for all such maps ${ }^{21}$, and not only for those of class $W^{1, h+1}$. The continuity of $J_{\psi}$ (and $J_{\omega}$ ) ensures that distributional and pointwise definition of $J_{\psi}$ agree for all bounded maps of class $W^{1, h+1}$ (and of course identity (7.2) holds too).

7.3. Integration of maps valued in a Banach space. Let $E$ be the dual of a separable Banach space, $X$ a subset of some euclidean space endowed with a finite positive measure $\mu$, and $f$ a map in $L_{*}^{1}(X, E)$ - that is, a Borel map from $X$ into $E$, endowed with the weak ${ }^{*}$ topology, such that $\|f\|_{1}:=\int_{X}|f|_{E} d \mu$ is finite. Then the integral of $f$ on $X$ is the element of $E$ defined by $\langle e, v\rangle=\int_{X}\langle f, v\rangle d \mu$ for every $v$ in the pre-dual of $E$.

We say that $f$ is approximately continuous in the weak* sense at $\bar{x} \in X$ if: (a) the function $x \mapsto|f(x)|_{E}$ is approximately continuous at $\bar{x},{ }^{22}$ and (b) the function $x \mapsto\langle f(x), v\rangle$ is approximately continuous at $\bar{x}$ for every $v$ in the predual of $E$. It is easy to see that in this case, the averages of $f(x)$ over $x \in B(\bar{x}, r)$ converge to $f(\bar{x})$ in the weak $*$ topology of $E$, and also their norms converge. More generally, the integrals $\int_{X} f \rho_{i} d \mu$ converge weakly* to $f(\bar{x})$ if the real functions $\rho_{i}$ converge to a Dirac mass at $\bar{x}$, and are sufficiently well-distributed around $\bar{x}$, e.g., for every $i$ there exists $r_{i}>0$ such that $\rho_{i}$ is supported in $B\left(x, r_{i}\right)$ and

21 The Jacobian $J u$ agrees with $J_{\psi} u=d J_{\omega_{0}} u$ where $\psi(y)=d y=d y_{1} \wedge \cdots \wedge d y_{k}$ is the standard volume form on $\mathbb{R}^{k}$, and $\omega_{0}$ is given in (2.7) divided by $k$.

22 Approximate continuity of $g$ at $\bar{x}$ is intended in the usual $L^{1}$ sense, namely that the average of $|g(x)-g(\bar{x})|$ over all $x \in B(\bar{x}, r)$ tends to 0 as $r \rightarrow 0$. 
$\left|\rho_{i}\right| \leq C / \mu\left(B\left(\bar{x}, r_{i}\right)\right)$ for some finite constant $C$. Note that every $f$ in $L_{*}^{1}(X, E)$ is approximately continuous in the weak* sense at almost every $\bar{x} \in X{ }^{23}$

In Sects. 7.4 and 7.5 we apply these notions when $E$ is the space of bounded measures on $\Omega$-dual of $C_{0}(\Omega)$, space of continuous functions vanishing at $\partial \Omega$ - or the space of $d$-dimensional currents with finite mass in $\Omega$-dual of $C_{0}\left(\Omega, \wedge^{d}\left(\mathbb{R}^{n}\right)\right)$, space of continuous $d$-forms vanishing at $\partial \Omega$. Notice that neither spaces are separable or have the Radon-Nikodym property, i.e., there are functions in $L_{*}^{1}(X, E)$ which cannot be integrated in the sense of Bochner and are nowhere approximately continuous in the usual (strong) sense.

\section{Oriented and unoriented coarea formula}

For the rest of this subsection $M$ is a smooth oriented $h$-surface in $\mathbb{R}^{k}$ without boundary, and $\omega$ is the standard volume form on $M$.

7.4. Coarea formula for Lipschitz maps. The usual coarea formula (see [17, Theorem 3.2.22]) says that for every Lipschitz map $u: \Omega \rightarrow M$, every Borel set $A \subset \Omega$, and every continuous function $\rho: M \rightarrow \mathbb{R}$ there holds

$$
\int_{A}\left|J_{\omega} u\right| \cdot|\rho(u)| d \mathcal{L}^{n}=\int_{M} \mathcal{H}^{n-h}\left(N_{y} \cap A\right) \cdot|\rho(y)| d \mathcal{H}^{h}(y),
$$

where $N_{y}$ is the level set $u^{-1}(y)$ for every $y \in M$. Moreover $N_{y}$ is $(n-h)$-rectifiable for $\mathcal{H}^{h}$-a.e. $y \in M$.

If $A$ is the complement of the set of all $x$ such that $u$ is differentiable at $x$ and $J_{\omega} u(x) \neq 0$, then the integral of $\left|J_{\omega} u\right|$ on $A$ vanishes, and applying formula (7.4) with $\rho(y) \equiv 1$ we deduce that, for $\mathcal{H}^{h}$-a.e. $y \in M$, the map $u$ is differentiable at $\mathcal{H}^{n-h}$-a.e. $x$ in $N_{y}$, and the $(n-h)$-vector $\star J_{\omega} u(x)$ does not vanish. Hence $\star J_{\omega} u(x)$ is a simple vector which spans the tangent space to $N_{y}$ at any such point $x$.

Now we equip each rectifiable level set $N_{y}$ with the orientation $\star J_{\omega} u /\left|\star J_{\omega} u\right|$ and the multiplicity 1 , and make it a rectifiable current with finite mass, still denoted by $N_{y}$. Since $|\omega|=1$ on $M$, for $\rho \equiv 1$ inequality (7.1) and formula (7.4) yield

$$
\int_{M}\left\|N_{y}\right\| d \mathcal{H}^{h}(y)=\int_{\Omega}\left|J_{\omega} u\right| d \mathcal{L}^{n} \leq \int_{\Omega}|D u|^{h} d \mathcal{L}^{n} .
$$

Hence, taking into account that $\rho(u) J_{\omega} u=J_{\rho \omega} u$, identity (7.4) can be rewritten as a decomposition of the measure $\left|\star J_{\rho \omega} u\right|$ as a weighted average over the parameter $y$ of the measures $\left|N_{y}\right|$, that is,

$$
\left|\star J_{\rho \omega} u\right|=\int_{M}\left|N_{y}\right| \cdot|\rho(y)| d \mathcal{H}^{h}(y),
$$

where the integral is intended in the sense of Sect. 7.3 (the map $y \mapsto\left|N_{y}\right|$ is summable by (7.5); we omit checking that it is also weak* Borel measurable).

\footnotetext{
23 Indeed, if $\bar{x}$ satisfies (a), then (b) holds for all $v$ in the pre-dual of $E$ if (and only if) it holds for all $v$ in a given countable dense subset of the pre-dual of $E$.
} 
From (7.6) we immediately derive the analogous decomposition for the current $\star J_{\rho \omega} u$ in terms of the currents $N_{y}$ (oriented coarea formula):

$$
\star J_{\rho \omega} u=\int_{M} N_{y} \cdot \rho(y) d \mathcal{H}^{h}(y) .
$$

7.5. Coarea formula for Sobolev maps. Formulas (7.4-7.7) can be extended with some care to maps $u$ in $W^{1, h}(\Omega, M)$. The key step is to establish a suitable version of (7.4).

We first choose finitely many smooth $h$-surfaces $M_{i}$, which are diffeomorphic to closed balls in $\mathbb{R}^{h}$ and cover $M$. Let now $E$ be the set of all points where $u$ is not approximately differentiable: then $E$ is Lebesgue negligible, and $\Omega \backslash E$ can be covered by a countably many sets $B_{j}$ where $u$ agrees with a Lipschitz map (cf. [17, Theorem 3.1.8]). We also require that the sets $B_{j}$ are pairwise disjoint and $u\left(B_{j}\right)$ is contained in one of the surfaces $M_{i}$.

Since each $M_{i}$ is diffeomorphic to a closed ball in $\mathbb{R}^{h}$, we can use Kirszbraun's extension theorem (for maps into a closed convex subset of $\mathbb{R}^{h}$ ) to find a Lipschitz map $u_{j}: \Omega \rightarrow M_{i}$ which agrees with $u$ on $B_{j}$. Thus formula (7.4) holds for $u_{j}$, and since $u$ agrees with $u_{j}$ in $B_{j}$ and $D u$ agrees with $D u_{j}$ a.e. in $B_{j}$, formula (7.4) holds also for $u$ provided $A \subset B_{j}$ for some $j$. As the sets $B_{j}$ cover $\Omega \backslash E$, (7.4) can be made true for every $u$ and every set $A \subset \Omega$ by re-defining $N_{y}$ as

$$
N_{y}:=u^{-1}(y) \backslash E \text { for every } y \in M .
$$

Since $u$ agrees with $u_{j}$ on each $B_{j}$, each $N_{y}$ is rectifiable for $\mathcal{H}^{k}$-a.e. $y \in M$ and $\star J_{\omega} u /\left|\star J_{\omega} u\right|$ is an orientation of $N_{y}$. Now we can proceed as in the previous paragraph and endow $N_{y}$ with the structure of rectifiable current, and show that the decomposition formulas (7.6) and (7.7), and estimate (7.5) hold.

Since $y \mapsto N_{y}$ is an $L_{*}^{1}$ map from $M$ into the Banach space of $(n-h)$-currents with finite mass, as pointed out in Sect. 7.3, it is approximately continuous in the weak* sense at $\mathcal{H}^{h}$-a.e. $y \in M$. We call such $y$ regular values, and the corresponding $N_{y}$ regular level sets. In the following we use the notation $N_{y}$ only for regular level sets.

Remark 7.6. Formula (7.4) holds also for maps $u$ of class $W^{1,1}$, provided that the level sets $N_{y}$ are defined as in (7.8), and $\left|J_{\omega} u\right|$ is defined in the pointwise sense. The assumption that $u$ is of class $W^{1, h}$ is used to prove that $\mathcal{H}^{n-k}\left(N_{y}\right)$ is finite for a.e. $y \in M$ and that the map $y \mapsto\left\|N_{y}\right\|$ is summable (cf. (7.5)), which in turn is needed to ensure that the integrals at the right-hand sides of (7.6) and (7.7) make sense (cf. Sect. 7.3).

Remark 7.7. If we define the level sets $N_{y}$ of a map $u \in W^{1,1}(\Omega, M)$ simply as $u^{-1}(y)-$ that is, we do not remove the points of non-differentiability of $u-$ then formula (7.4) remains valid provided that $u$ satisfies the following version of the (N) property: for every null set $E \subset \mathbb{R}^{n}, \mathcal{H}^{n-h}\left(u^{-1}(y) \cap E\right)=0$ for $\mathcal{H}^{h}$-a.e. $y \in M$. Formula (7.4), with $A$ replaced by $E$, shows that every Lipschitz map $u$ satisfies the $(\mathrm{N})$ property. It has been recently proved in [30] that the same is true when $u$ is the precise representative of a map of class $W^{1, p}$ for some $p>h$. 
Remark 7.8. If $M$ is the boundary of an $(h+1)$-surface $M^{\prime}$ and $u$ is sufficiently regular, and precisely of class $W^{1, h+1}$, then $\partial N_{y}=0$ for every regular value $y \in M$. Indeed, taken any extension of the form $\omega$ to $M^{\prime}$, formulas (7.7) and (2.2) yield, for every smooth function $\rho$,

$$
\int_{M} \partial N_{y} \cdot \rho(y) d \mathcal{H}^{h}(y)=\partial\left(\star J_{\rho \omega} u\right)=(-1)^{n-h} \star J_{d(\rho \omega)} u,
$$

and since $u$ takes values in a negligible subset of $M^{\prime}$, the $(h+1)$-form $J_{d(\rho \omega)} u$ - the pull-back of $d(\rho \omega)$ according to $u$ - must vanish. Thus the integral in the formula above vanishes for every $\rho$, which proves that $\partial N_{y}=0$ for a.e. $y \in M$, and by continuity for every regular value $y$.

\section{Proof of Theorem 3.8}

The geometric idea behind the proof of Theorem 3.8 is very simple: if we assume that $u$ is smooth outside a singular set $S$ of codimension $k$, then, up to a factor $\alpha_{k}$ and some integer multiplicity $\sigma$, the current $\star J u$ agrees with $S$ (see Sect. 3.7), and, as shown in Fig. 6 below, $S$ is the boundary of every level set $N_{y}:=u^{-1}(y)$, which is indeed a surface of codimension $k-1$.

We claim that the same is true, in some sense, also for maps in $W^{1, k-1}$. The key observation is that not only $J u=\frac{1}{k} d\left(J_{\omega_{0}} u\right)$ where $\omega_{0}$ is the standard volume form on $S^{k-1}$, but in fact $J u=\frac{1}{k} d\left(J_{\rho \omega_{0}} u\right)$ for any smooth function $\rho$ with average one (Proposition 7.9). Therefore we can use the coarea formula to represent $\star J u$ as the boundary of the integral combination $\int N_{y} \rho(y) d \mathcal{H}^{k-1}(y)$, and then pass to the limit as the functions $\rho$ converge to a Dirac's mass at some regular value $y$.

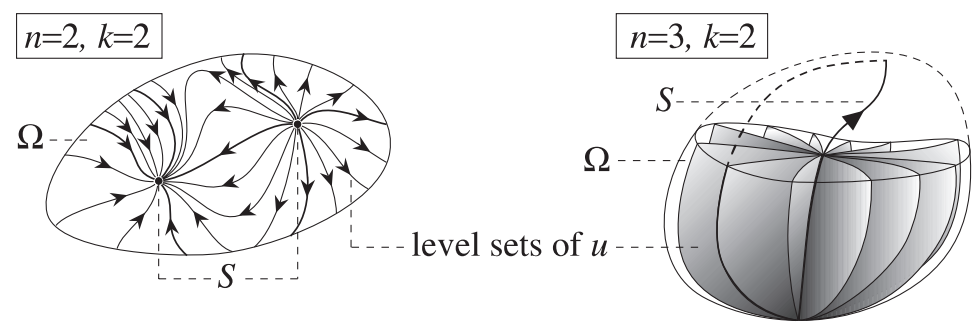

Fig. 6 .

In the following statement we consider a compact, oriented $h$-surface $M$ without boundary. Notice that in this case any smooth $h$-form on $M$ can be written as $\rho \omega$ where $\omega$ is the standard volume form on $M$, and $\rho$ is a real function on $M$.

Proposition 7.9. If $M$ is connected and $\rho: M \rightarrow \mathbb{R}$ is a smooth function with average 1 , then

$$
d\left(J_{\omega} u\right)=d\left(J_{\rho \omega} u\right) \quad \text { for every } u \in W^{1, h}(\Omega, M) .
$$


Proof. It suffices to show that $J_{\omega} u-J_{\rho \omega} u=J_{(1-\rho) \omega} u$ is the differential of an $(h-1)$-form.

The $h$-form $(1-\rho) \omega$ has vanishing integral over $M$, and since the $h$-th De Rham cohomology group of a connected $h$-dimensional manifold $M$ is $\mathbb{R}$, and the cohomology class of an $h$-form is determined by the integral over $M$ (cf. [10, Corollary 5.8]), then $(1-\rho) \omega$ belongs to the class 0 , i.e., it is an exact form. Thus there exists an $(h-1)$-form $\varphi$ on $M$ such that $d \varphi=(1-\rho) \omega$. If we extend $\varphi$ to the rest of $\mathbb{R}^{k}$ in an arbitrary way, then $d \varphi$ is an extension of $(1-\rho) \omega$, and for every $u$ of class $W^{1, h}$ there holds (cf. (7.2))

$$
J_{(1-\rho) \omega} u=J_{d \varphi} u=d J_{\varphi} u
$$

Proof of Theorem 3.8. By Definition 3.2, the Jacobian of $u$ is $J u=\frac{1}{k} d\left(J_{\omega_{0}} u\right)$, where $\omega_{0}$ is the standard volume-form $S^{k-1}$. Let $\rho$ be a smooth function on $S^{k-1}$ with average 1 , that is, with integral $k \alpha_{k}$. Then, $J u=\frac{1}{k} d\left(J_{\rho \omega} u\right)$ by Proposition 7.9, and recalling (2.2),

$$
\star J u=(-1)^{n-k+1} \frac{1}{k} \partial\left(\star J_{\rho_{0} \omega} u\right) .
$$

As discussed in Sect. 7.5, decomposition formula (7.7) applies to $\star J_{\rho \omega_{0}} u$, too, and therefore (7.10) becomes

$$
\star J u=(-1)^{n-k+1} \frac{1}{k} \partial\left[\int_{S^{k-1}} N_{y} \cdot \rho(y) d \mathcal{H}^{k-1}(y)\right],
$$

where the currents $N_{y}$ are the $y$-level set of $u$, as defined in Sect. 7.5.

If $\bar{y}$ is a regular value of $u$, namely a point of approximate continuity (in the weak* sense) of the map $y \mapsto N_{y}$, and we properly choose a sequence of smooth functions $\rho$ which converge to the Dirac's mass $k \alpha_{k} \delta_{\bar{y}}$, then the integral between square brackets at the right-hand side of (7.11) converge in the sense of currents to $k \alpha_{k} N_{\bar{y}}$ (cf. Sect. 7.3), and then

$$
\star J u=(-1)^{n-k+1} \alpha_{k} \partial N_{\bar{y}} .
$$

If in addition we have chosen $\bar{y}$ so that $\left\|N_{\bar{y}}\right\|$ is smaller than the average of $\left\|N_{y}\right\|$ over all $y \in S^{k-1}$, inequality (7.5) yields (3.7).

Remark 7.10. The key lemma in the proof of Theorem 3.8, namely Proposition 7.9, is a rephrasing of a known fact on the structure of the boundary of graphs with finite area. Let $u$ be a map in $W^{1, h}(\Omega, M)$, and let $G_{u}$ be the regular part of the graph of $u$, namely, the set of all $(x, u(x))$ such that $u$ is approximately continuous and approximately differentiable at $x$. One easily checks that $G_{u}$ is an $n$-rectifiable set with finite measure (and according to [20, §3.2.1], we write $u \in \mathcal{A}_{1}(\Omega, M)$ ), and the canonical orientation induced by the projection on $\Omega$ makes it a rectifiable $n$-current. Moreover, if $M$ is connected, $\partial G_{u}$ can be represented as a product $T \times M$, where $T$ is a current of dimension $n-h-1$ in $\Omega$ (see, e.g., [21, §4.2.1], for a special case), and using the coarea formula one can prove that $T$ remains unchanged if we 
replace the volume form on $M$ by any other form with same integral. If $M=S^{k-1}$, the relation with the Jacobian is given by the identity $\star J u=\frac{1}{k}\left(\pi_{\sharp}\left(\partial G_{u} \mathrm{~L} \omega_{0}\right)\right)$, where $\pi$ is the projection of $\Omega \times S^{k-1}$ onto $\Omega$, and $\omega_{0}$ is the $(k-1)$-form in (2.7). In particular $\star J u=\alpha_{k} T$.

\section{Approximation of rectifiable currents}

The main result in this subsection is Theorem 7.12 below, which is a fairly simple consequence of Theorem 4.2.22 in [17].

Definition 7.11. Given two rectifiable h-currents $N_{1}$ and $N_{2}$ in $\Omega$, we consider the distance $d\left(N_{1}, N_{2}\right)$ defined by

$$
d\left(N_{1}, N_{2}\right):=\inf (\|R\|+\|P\|)
$$

where the infimum is taken over all rectifiable currents $R$ and $P$ of dimension $h$ and $h+1$, respectively, such that $N_{1}-N_{2}=R+\partial P .{ }^{24}$ In the following we say that a sequence of rectifiable currents $N_{i}$ converge in the flat metric with convergence of the masses to $N$ if $d\left(N_{i}, N\right) \rightarrow 0$ and $\left\|N_{i}\right\| \rightarrow\|N\|$.

Theorem 7.12. Let $N$ be an h-dimensional rectifiable current in $\Omega$ with finite mass. Then $N$ can be approximated in the flat metric with convergence of masses by finite sums $\sum E_{i}$, where each $E_{i}$ is an oriented h-dimensional disk with center $x_{i}$ and radius $r_{i}$, endowed with constant multiplicity 1 .

Moreover, given a function $\rho$ on $\Omega$ which is strictly positive $\mathcal{H}^{h}$-a.e., we may require that the radii $r_{i}$ satisfy $r_{i}<\rho\left(x_{i}\right)$, and the balls $\bar{B}\left(x_{i}, r_{i}\right)$ are pairwise disjoint and contained in $\Omega$.

Proof. By Theorem 4.2.22 in [17], there is no loss of generality in assuming that $N$ is a polyhedral current compactly supported in $N$.

Moreover, we may also assume that the multiplicity of $N$ is 1 on each face of the polyhedron. Indeed, if some face of $N$ was initially assigned a multiplicity $\sigma>0$, it can be replaced by $\sigma$ copies of itself (where each of the copies is translated by a small vector, and equipped with multiplicity 1 ): this operation does not change the mass of $N$, and the resulting current can be made arbitrarily close to $N$ in the flat distance.

To prove the theorem for this special class of currents, it suffices to use Besicovitch covering theorem to cover each face $S$ of $N$ with finitely many pairwise disjoint disks $E_{i}$ of center $x_{i}$ and radius $r_{i}$ which cover all of $S$ except a subset with small measure and satisfy $r_{i}<\bar{\rho}\left(x_{i}\right)$, where $\bar{\rho}(x)$ is the minimum between $\rho(x)$ and half the distance between $x$ and $N \backslash S$. This choice of $\bar{\rho}$ implies the second part of the statement.

Corollary 7.13. Let $N$ be an h-dimensional rectifiable current in $\Omega$ with finite mass, and $\rho$ a function on $\Omega$ which is strictly positive $\mathcal{H}^{h}$-a.e. Then there exist finitely many oriented $h$-disks $E_{i}$ with centers $x_{i}$ and radii $r_{i}<\rho\left(x_{i}\right)$, rectifiable currents $R$ and $P$ of dimension $h$ and $h+1$, respectively, so that

24 Thus $d$ is a modification of the usual flat metric - see [17, §4.1.12] or [35, §31]. 
(i) $N=\sum_{i} E_{i}+R+\partial P$;

(ii) $\sum_{i}\left\|E_{i}\right\| \leq 2^{1-h}\|N\|$;

(iii) $\|R\|+\|P\| \leq\left(1-2^{-1-h}\right)\|N\|$;

(iv) the balls $\bar{B}\left(x_{i}, 2 r_{i}\right)$ are pairwise disjoint.

Proof. Let $\varepsilon>0$ be fixed for the time being (to be properly chosen later). By Theorem 7.12 (and Definition 7.11) we can find disjoint disks $E_{i}^{\prime}$, and rectifiable currents $R^{\prime}$ and $P^{\prime}$ such that

$$
N=\sum_{i} E_{i}^{\prime}+R^{\prime}+\partial P^{\prime}, \sum_{i}\left\|E_{i}^{\prime}\right\| \leq(1+\varepsilon)\|N\|,\left\|R^{\prime}\right\|+\left\|P^{\prime}\right\| \leq \varepsilon\|N\| .
$$

Let now $E_{i}$ be a copy of $E_{i}^{\prime}$ scaled by a factor $1 / 2$ and concentric to $E_{i}^{\prime}, P:=P^{\prime}$ and $R:=\sum\left(E_{i}^{\prime}-E_{i}\right)+R^{\prime}$. Then the first identity in (7.13) yields (i), the first inequality in (7.13) yields (ii) (at least for for $\varepsilon \leq 1$ ), and

$$
\begin{aligned}
\|R\|+\|P\| & \leq \sum_{i}\left\|E_{i}^{\prime}-E_{i}\right\|+\left\|R^{\prime}\right\|+\left\|P^{\prime}\right\| \\
& \leq\left(1-2^{-h}\right) \sum_{i}\left\|E_{i}^{\prime}\right\|+\varepsilon\|N\| \\
& \leq\left[\left(1-2^{-h}\right)(1+\varepsilon)+\varepsilon\right]\|N\|,
\end{aligned}
$$

which gives (iii) if we choose $\varepsilon$ small enough to have $\left(1-2^{-h}\right)(1+\varepsilon)+\varepsilon \leq$ $1-2^{-h-1}$. Finally, if the disks $E_{i}^{\prime}$ satisfy the second part of Theorem 7.12, then (iv) holds.

\section{References}

1. Alberti, G.: Un risultato di convergenza variazionale per funzionali di tipo GinzburgLandau in dimensione qualunque (A variational convergence result for functionals of Ginzburg-Landau type in any dimension). Boll. Unione Mat. Ital., Sez. B, Artic. Ric. Mat. (8) 4, 289-310 (2001)

2. Alberti, G., Baldo, S., Orlandi, G.: Variational convergence for functionals of GinzburgLandau type. Preprint 2002

3. Ambrosio, L., Fusco, N., Pallara, D.: Functions of bounded variation and free discontinuity problems. Oxford Mathematical Monographs. Oxford: Oxford Science Publications 1999

4. Baldo, S., Orlandi, G.: A note on the Hodge theory for functionals with linear growth. Manuscr. Math. 97, 453-467 (1998)

5. Ball, J.M.: Convexity conditions and existence theorems in nonlinear elasticity. Arch. Ration. Mech. Anal. 63, 337-403 (1977)

6. Bethuel, F.: The approximation problem for Sobolev maps between two manifolds. Acta Math. 167, 153-206 (1991)

7. Bethuel, F., Coron, J.-M., Demengel, F., Hélein, F.: A cohomological criterion for density of smooth maps in Sobolev spaces between two manifolds. Nematics. Mathematical and physical aspects (Orsay, 1990), 15-23, ed. by J.-M. Coron et al. NATO Adv. Sci. Inst. Ser. C Math. Phys. Sci., 332. Dordrecht: Kluwer Academic Publishers 1991

8. Bethuel, F., Demengel, F.: Extensions of Sobolev mappings between manifolds. Calc. Var. Partial Differ. Equ. 3, 375-391 (1995) 
9. Bochnak, J., Kucharz, W.: Complete intersections in differential topology and analytic geometry. Boll. Unione Mat. Ital., Sez. B, Artic. Ric. Mat. (7) 10, 1019-1041 (1996)

10. Bott, R., Tu, L.W.: Differential forms in algebraic topology. Graduate Texts in Mathematics, 82. New York, Berlin: Springer 1982

11. Bourgain, J., Brezis, H., Mironescu, P.: Lifting in Sobolev spaces. J. Anal. Math. 80, 37-86 (2000)

12. Bourgain, J., Brezis, H., Mironescu, P.: On the structure of the Sobolev space $H^{1 / 2}$ with values into the circle. C. R. Acad. Sci., Paris, Sér. I, Math. 331, 119-124 (2000)

13. Bredon, G.E.: Topology and geometry. Corrected third printing of the 1993 original. Graduate Texts in Mathematics, 139. New York: Springer 1997

14. Brezis, H., Coron, J.-M., Lieb, E.: Harmonic maps with defects. Commun. Math. Phys. 107, 649-705 (1986)

15. Demengel, F.: Une caractérisation des applications de $W^{1,1}\left(B^{n}, S^{1}\right)$ qui peuvent être approchées par des fonctions régulières [A characterization of maps in $W^{1,1}\left(B^{n}, S^{1}\right)$ that can be approximated by smooth maps]. C. R. Acad. Sci., Paris, Sér. I, Math. 310, 553-557 (1990)

16. Evans, L.C., Gariepy, R.F.: Measure theory and fine properties of functions. Studies in Advanced Mathematics. Boca Raton: CRC Press 1992

17. Federer, H.: Geometric measure theory. Grundlehren der mathematischen Wissenschaften, 153. New York: Springer 1969. Reprinted in the series Classics in Mathematics. Berlin, Heidelberg: Springer 1996

18. Gagliardo, E.: Caratterizzazione delle traccie sulla frontiera relativa ad alcune classi di funzioni in piú variabili. Rend. Semin. Mat. Univ Padova 27, 284-305 (1957)

19. Giaquinta, M., Modica, G., Souček, J.: Cartesian currents, weak diffeomorphism, and existence theorems in nonlinear elasticity. Arch. Ration. Mech. Anal. 106, 97-159 (1989). Erratum and addendum, Arch. Ration. Mech. Anal. 109, 385-392 (1990)

20. Giaquinta, M., Modica, G., Souček, J.: Cartesian currents in the calculus of variations. I. Cartesian currents. Ergebnisse der Mathematik und ihrer Grenzgebiete. 3. Folge, 37. Berlin: Springer 1998

21. Giaquinta, M., Modica, G., Souček, J.: Cartesian currents in the calculus of variations. II. Variational integrals. Ergeb. Math. Grenzgeb., 3. Folge, 38. Berlin: Springer 1998

22. Guillemin, V., Pollack, A.: Differential topology. Englewood Cliffs, N.J.: Prentice-Hall 1974

23. Hang, F.-B., Lin, F.-H.: A remark on the Jacobians. Commun. Contemp. Math. 2, 35-46 (2000)

24. Hardt, R., Rivière, T.: Connecting topological Hopf singularities. To appear in Ann. Scuola Norm. Sup. Pisa

25. Hirsch, M.W.: Differential topology. Corrected reprint of the 1976 original. Graduate Texts in Mathematics, 33. New York: Springer 1994

26. Jerrard, R.L.: A new proof of the rectifiable slices theorem. Ann. Scuola Norm. Sup. Pisa (5) 1, 905-924 (2002)

27. Jerrard, R.L., Soner, H.M.: Rectifiability of the distributional Jacobian for a class of functions. C. R. Acad. Sci., Paris, Sér. I, Math. 329, 683-688 (1999)

28. Jerrard, R.L., Soner, H.M.: Functions of bounded hi gher variation. Indiana Univ. Math. J. 51, 645-677 (2002)

29. Jerrard, R.L., Soner, H.M.: The Jacobian and the Ginzburg-Landau energy. Calc. Var. Partial Differ. Equ. 14, 151-191 (2002)

30. Malý, J., Swanson, D., Ziemer, W.P.: The coarea formula for Sobolev mappings. Trans. Am. Math. Soc. 355, 477-492 (2003)

31. Müller, S.: Det=det. A remark on the distributional determinant. C. R. Acad. Sci., Paris, Sér. I, Math. 311, 13-17 (1990)

32. Pakzad, M.R.: On topological singular set of maps with finite 3-energy into $S^{3}$.Z. Anal. Anwend. 21, 529-530 (2002)

33. Pakzad, M.R., Rivière, T.: Weak density of smooth maps for the Dirichlet energy between manifolds. Geom. Funct. Anal. 13, 223-257 (2003) 
34. Rivière, T.: Dense subsets of $H^{1 / 2}\left(S^{2}, S^{1}\right)$. Ann. Global Anal. Geom. 18, 517-528 (2000)

35. Simon, L.: Lectures on geometric measure theory. Proceedings of the Centre for Mathematical Analysis 3. Canberra: Australian National University 1983

36. Steenrod, N.: The topology of fibre bundles. Princeton Mathematical Series vol. 14. Princeton: Princeton University Press 1951. Reprinted in the series Princeton Landmarks in Mathematics. Princeton: Princeton University Press 1999

37. White, B.: Rectifiability of flat chains. Ann. Math. 150, 165-184 (1999) 